\title{
On Third Order Stable Difference Scheme for Hyperbolic Multipoint Nonlocal Boundary Value Problem
}

\author{
Ozgur Yildirim and Meltem Uzun \\ Department of Mathematics, Yildiz Technical University, Esenler, 34210 Istanbul, Turkey \\ Correspondence should be addressed to Ozgur Yildirim; ozgury@yildiz.edu.tr
}

Received 31 October 2014; Accepted 18 May 2015

Academic Editor: Binggen Zhang

Copyright ( 2015 O. Yildirim and M. Uzun. This is an open access article distributed under the Creative Commons Attribution License, which permits unrestricted use, distribution, and reproduction in any medium, provided the original work is properly cited.

This paper presents a third order of accuracy stable difference scheme for the approximate solution of multipoint nonlocal boundary value problem of the hyperbolic type in a Hilbert space with self-adjoint positive definite operator. Stability estimates for solution of the difference scheme are obtained. Some results of numerical experiments that support theoretical statements are presented.

\section{Introduction}

Hyperbolic partial differential equations (PDEs) are of growing interest in several areas of engineering and natural sciences such as acoustic, electromagnetic, hydrodynamic, elasticity, fluid mechanics, and other areas of physics (see, e.g., [1-5] and the references given therein). In some cases, classical boundary conditions cannot describe process or phenomenon precisely. Therefore, mathematical models of various physical, chemical, biological, or environmental processes often involve nonclassical conditions. Such conditions are usually identified as nonlocal boundary conditions and project situations when the data on the domain boundary cannot be measured directly or when the data on the boundary depend on the data inside the domain. Many researchers study nonlocal boundary value problems (NBVPs) for hyperbolic and mixed types of partial differential equations. There are many results (see [6-10]) on integral inequalities with two dependent limits to theory of integral-differential equations of the hyperbolic type and of difference schemes for the approximate solution of these problems. Stability has been actively studied in the development of numerical methods for the approximate solutions of PDEs (see [5-31]). In particular, an appropriate model for the analysis of stability is provided by a suitable unconditionally stable difference scheme with an unbounded operator.
It is known (see $[29,30])$ that various multipoint NBVPs for hyperbolic equations can be reduced to the problem

$$
\begin{aligned}
\frac{d^{2} u(t)}{d t^{2}}+A u(t) & =f(t) \quad 0 \leq t \leq 1, \\
u(0) & =\sum_{r=1}^{n} \alpha_{r} u\left(\lambda_{r}\right)+\varphi, \\
u_{t}(0) & =\sum_{r=1}^{n} \beta_{r} u_{t}\left(\lambda_{r}\right)+\psi, \\
& \quad 0<\lambda_{1}<\lambda_{2}<\cdots<\lambda_{n} \leq 1 .
\end{aligned}
$$

Here $A$ is a self-adjoint positive definite operator with domain $D(A)$ in a Hilbert space $H$.

A function $u(t)$ is called a solution of problem (1) if the following conditions are satisfied:

(i) $u(t)$ is twice continuously differentiable on the segment $[0,1]$. The derivatives at the endpoints of the segment are understood as the appropriate unilateral derivatives.

(ii) The element $u(t)$ belongs to $D(A)$ for all $t \in[0,1]$ and the function $A u(t)$ is continuous on the segment $[0,1]$.

(iii) $u(t)$ satisfies the equations and the initial conditions (1). 
In the last decades, many scientists worked and published scientific papers in the field of finite difference method for the numerical solutions of hyperbolic PDEs. In this field, first order and two types of second order stable difference schemes for the solution of abstract hyperbolic problem (1) were presented in [17] and high order of accuracy difference schemes for the solution of the same problem were presented in [18]. However, the difference schemes presented in these references are generated by square roots of $A$. This action is not good for realization. Therefore, in spite of theoretical results the role of their application to a numerical solution of (1) is not great.

In the present paper, a third order of accuracy unconditionally stable difference scheme generated by the integer power of $A$ for the approximate solution of the multipoint NBVP (1) is presented. Stability estimates for the solution of this difference scheme are established. [16].

Some results of this paper, without proof, are published in

Note that many scientists have studied the solutions of boundary value problems such as parabolic equations, elliptic equations, and equations of mixed types extensively (see, e.g., $[6-10,21-31]$ and the references therein).

\section{Third-Order Accurate Stable Difference Scheme}

In the present section a third order of accuracy stable difference scheme for the approximate solution of multipoint NBVP (1) is presented. We associate problem (1) with the corresponding third order of accuracy difference scheme:

$$
\begin{aligned}
& \tau^{-2}\left(u_{k+1}-2 u_{k}+u_{k-1}\right)+\frac{2}{3} A u_{k}+\frac{1}{6} A\left(u_{k+1}+u_{k-1}\right) \\
&+\frac{1}{12} \tau^{2} A^{2} u_{k+1}=f_{k}, \\
& f_{k}=\frac{2}{3} f\left(t_{k}\right)+\frac{1}{6}\left(f\left(t_{k+1}\right)+f\left(t_{k-1}\right)\right)-\frac{1}{12} \\
& \cdot \tau^{2}\left(-A f\left(t_{k+1}\right)+f^{\prime \prime}\left(t_{k+1}\right)\right) \\
&\left(I-i \tau A^{2}\right) u_{0}=\sum_{k=1}^{n} \alpha_{k}\left\{u_{\left[\lambda_{k} / \tau\right]}=k \tau, 1 \leq k \leq N-1, N \tau=1\right. \\
&+ \\
&+\tau^{-1}\left(u_{\left[\lambda_{k} / \tau\right]}-u_{\left[\lambda_{k} / \tau\right]-1}\right)\left(\lambda_{k}-\left[\frac{\lambda_{k}}{\tau}\right] \tau\right) \\
&+\frac{3}{2}\left(f_{\left[\lambda_{k} / \tau\right]}-A u_{\left[\lambda_{k} / \tau\right]}\right)\left(\lambda_{k}-\left[\frac{\lambda_{k}}{\tau}\right] \tau\right)^{2} \\
&+\frac{7}{6}\left(f_{\left[\lambda_{k} / \tau\right]}^{\prime}-\tau^{-1} A\left(u_{\left[\lambda_{k} / \tau\right]}-u_{\left[\lambda_{k} / \tau\right]-1}\right)\right)
\end{aligned}
$$

$$
\begin{aligned}
&\left.\left(\lambda_{k}-\left[\frac{\lambda_{k}}{\tau}\right] \tau\right)^{3}\right\}+\varphi \\
&\left(I+i \tau A^{2}\right) \tau^{-1}\left(u_{1}-u_{0}\right)=\sum_{k=1}^{n} \beta_{k} \\
& \cdot\left\{\tau^{-1}\left(u_{\left[\lambda_{k} / \tau\right]}-u_{\left[\lambda_{k} / \tau\right]-1}\right)+\left(f_{\left[\lambda_{k} / \tau\right]}-A u_{[\lambda k / \tau]}\right)\right. \\
& \cdot\left(\lambda_{k}-\left[\frac{\lambda_{k}}{\tau}\right] \tau\right) \\
&+\frac{1}{2 !}\left(f_{\left[\lambda_{k} / \tau\right]}^{\prime}-\tau^{-1} A\left(u_{\left[\lambda_{k} / \tau\right]}-u_{\left[\lambda_{k} / \tau\right]-1}\right)\right) \\
& \cdot\left(\lambda_{k}-\left[\frac{\lambda_{k}}{\tau}\right] \tau\right)^{2} \\
&+\frac{1}{3 !}\left(f_{\left[\lambda_{k} / \tau\right]}^{\prime \prime}-A f_{\left[\lambda_{k} / \tau\right]}+A^{2} u_{\left[\lambda_{k} / \tau\right]}\right) \\
&\left..\left(\lambda_{k}-\left[\frac{\lambda_{k}}{\tau}\right] \tau\right)^{3}\right\}+\psi+f_{1,1} \tau .
\end{aligned}
$$

Here

$$
f_{1,1}=\left\{f(0)+\left(-f(0)+\tau f^{\prime}(0)\right) \frac{1}{2}-2 f^{\prime}(0) \frac{\tau}{6}\right\} .
$$

Note that throughout this paper for clarity $\lambda_{1}>2 \tau$ and $\lambda_{n}<1$ will be considered.

We are interested in studying the stability of solution of difference scheme (2) under assumption

$$
\begin{aligned}
& \sum_{k=1}^{n}\left|\alpha_{k}\right|\left\{1+\left|\frac{\lambda_{k}}{\tau}-\left[\frac{\lambda_{k}}{\tau}\right]\right|+\frac{3}{2}\left|\frac{\lambda_{k}}{\tau}-\left[\frac{\lambda_{k}}{\tau}\right]\right|^{2}\right. \\
& \left.+\frac{7}{6}\left|\frac{\lambda_{k}}{\tau}-\left[\frac{\lambda_{k}}{\tau}\right]\right|^{3}\right\}+\sum_{k=1}^{n}\left|\beta_{k}\right|\left\{1+\left|\frac{\lambda_{k}}{\tau}-\left[\frac{\lambda_{k}}{\tau}\right]\right|\right. \\
& \left.+\frac{1}{2}\left|\frac{\lambda_{k}}{\tau}-\left[\frac{\lambda_{k}}{\tau}\right]\right|^{2}+\frac{1}{6}\left|\frac{\lambda_{k}}{\tau}-\left[\frac{\lambda_{k}}{\tau}\right]\right|^{3}\right\}+\frac{1}{2} \sum_{k=1}^{n}\left|\alpha_{k}\right| \\
& \cdot \sum_{k=1}^{n}\left|\beta_{k}\right|\left\{1+\left|\frac{\lambda_{k}}{\tau}-\left[\frac{\lambda_{k}}{\tau}\right]\right|^{2}+\frac{7}{12}\left|\frac{\lambda_{k}}{\tau}-\left[\frac{\lambda_{k}}{\tau}\right]\right|^{4}\right. \\
& \left.+\frac{7}{36}\left|\frac{\lambda_{k}}{\tau}-\left[\frac{\lambda_{k}}{\tau}\right]\right|^{6}\right\}<1 .
\end{aligned}
$$


Throughout this section for simplicity we denote

$$
\begin{aligned}
B_{n}^{\tau} & =\left(I+\tau^{2} A^{4}\right)^{-1}\left\{1-\frac{1}{2} \sum_{k=1}^{n} \alpha_{k}\left(\left(\widetilde{R}_{1} R^{\left[\lambda_{k} / \tau\right]-1}-R_{1} \widetilde{R}^{\left[\lambda_{k} / \tau\right]-1}\right)\left(-\frac{1}{\tau}\left(\lambda_{k}-\left[\frac{\lambda_{k}}{\tau}\right] \tau\right)+\frac{7}{6 \tau} A\left(\lambda_{k}-\left[\frac{\lambda_{k}}{\tau}\right] \tau\right)^{3}\right)\right.\right. \\
& \left.+\left(\widetilde{R}_{1} R^{\left[\lambda_{k} / \tau\right]}-R_{1} \widetilde{R}^{\left[\lambda_{k} / \tau\right]}\right)\left(1+\frac{1}{\tau}\left(\lambda_{k}-\left[\frac{\lambda_{k}}{\tau}\right] \tau\right)-\frac{3}{2} A\left(\lambda_{k}-\left[\frac{\lambda_{k}}{\tau}\right] \tau\right)^{2}-\frac{7}{6 \tau} A\left(\lambda_{k}-\left[\frac{\lambda_{k}}{\tau}\right] \tau\right)^{3}\right)\right)-\frac{1}{2} \\
& \cdot R_{2} \sum_{k=1}^{n} \beta_{k}\left(\left(\widetilde{R}^{\left[\lambda_{k} / \tau\right]-1}-R^{\left[\lambda_{k} / \tau\right]-1}\right)\left(\frac{1}{\tau}-A\left(\lambda_{k}-\left[\frac{\lambda_{k}}{\tau}\right] \tau\right)-\frac{1}{2 \tau} A\left(\lambda_{k}-\left[\frac{\lambda_{k}}{\tau}\right] \tau\right)^{2}+\frac{1}{6} A^{2}\left(\lambda_{k}-\left[\frac{\lambda_{k}}{\tau}\right] \tau\right)^{3}\right)\right. \\
& \left.+\left(\widetilde{R}^{\left[\lambda_{k} / \tau\right]}-R^{\left[\lambda_{k} / \tau\right]}\right)\left(\frac{1}{\tau}-A\left(\lambda_{k}-\left[\frac{\lambda_{k}}{\tau}\right] \tau\right)-\frac{1}{2 \tau} A\left(\lambda_{k}-\left[\frac{\lambda_{k}}{\tau}\right] \tau\right)^{2}+\frac{1}{6} A^{2}\left(\lambda_{k}-\left[\frac{\lambda_{k}}{\tau}\right] \tau\right)^{3}\right)\right)+\frac{1}{4} \\
& \cdot R_{2} \sum_{k=1}^{n} \sum_{k=1}^{n} \alpha_{k} \beta_{k}\left(-\frac{1}{\tau}+A \tau\left(\lambda_{k}-\left[\frac{\lambda_{k}}{\tau}\right] \tau\right)^{2}+\frac{7}{12} A^{2} \tau^{3}\left(\lambda_{k}-\left[\frac{\lambda_{k}}{\tau}\right] \tau\right)^{4}-\frac{7}{36} A^{3} \tau^{5}\left(\lambda_{k}-\left[\frac{\lambda_{k}}{\tau}\right] \tau\right)^{6}\right)\left(\widetilde{R}_{1}-R_{1}\right) \\
& \left.\cdot\left[\widetilde{R}^{\left[\lambda_{k} / \tau\right]} R^{\left[\lambda_{k} / \tau\right]-1}(R-I)-R^{\left[\lambda_{k} / \tau\right]} \widetilde{R}^{\left[\lambda_{k} / \tau\right]-1}(\widetilde{R}-I)\right]\right\} .
\end{aligned}
$$

First, let us present some lemmas. We will consider the following operators:

$$
\begin{aligned}
R= & \left(I-\frac{1}{3} \tau^{2} A+i \tau A^{1 / 2} \sqrt{I+\frac{1}{72} \tau^{4} A^{2}}\right) \\
& \cdot\left(I+\frac{1}{6} \tau^{2} A+\frac{1}{12} \tau^{4} A^{2}\right)^{-1}
\end{aligned}
$$

and their conjugate $\widetilde{R}$

$$
\begin{aligned}
R_{1} & =\left(-\frac{5 \tau^{4}}{144} A^{2}+\frac{7 \tau^{6}}{216} A^{3}\right. \\
& \left.-i \tau A^{1 / 2}\left(I+\frac{\tau^{2}}{12} A+\frac{\tau^{4}}{144} A^{2}\right) \sqrt{I+\frac{1}{72} \tau^{4} A^{2}}\right) \\
& \cdot\left(-i \tau A^{1 / 2}\left(\sqrt{I+\frac{1}{72} \tau^{4} A^{2}}\right)\right. \\
& \left.\cdot\left(I+\frac{\tau^{2}}{12} A+\frac{\tau^{4}}{144} A^{2}\right)\right)^{-1},
\end{aligned}
$$

and their conjugate $\widetilde{R}_{1}$,

$$
\begin{aligned}
R_{2} & =\left(I-\frac{\tau^{2}}{12} A\right)\left(I+\frac{\tau^{2}}{6} A+\frac{\tau^{4}}{12} A^{2}\right) \\
& \left(-i A^{1 / 2}\left(I+\frac{\tau^{2}}{12} A+\frac{\tau^{4}}{144} A^{2}\right) \sqrt{I+\frac{1}{72} \tau^{4} A^{2}}\right)^{-1},
\end{aligned}
$$

$$
\begin{aligned}
R_{3} & =\left(I+\frac{\tau^{2}}{6} A+\frac{\tau^{4}}{12} A^{2}\right)\left(\left(I+\frac{\tau^{2}}{12} A+\frac{\tau^{4}}{144} A^{2}\right)\right. \\
\cdot & \left.\left(-i \tau A^{1 / 2} \sqrt{I+\frac{1}{72} \tau^{4} A^{2}}\right)\right)^{-1}, \\
R_{4} & =\left(I+\frac{\tau^{2}}{3} A+\frac{\tau^{4}}{9} A^{2}+\frac{\tau^{6}}{72} A^{3}\right) \\
& \left(-i A^{1 / 2}\left(\sqrt{I+\frac{1}{72} \tau^{4} A^{2}}\right)\left(I+\frac{\tau^{2}}{6} A+\frac{\tau^{4}}{12} A^{2}\right)\right. \\
& \left.\cdot\left(I+\frac{\tau^{2}}{6} A\right)\right)^{-1}, \\
R_{5} & =\left(-\frac{\tau^{2}}{2} A-\frac{\tau^{4}}{12} A^{2}+i \tau A^{1 / 2} \sqrt{I+\frac{1}{72} \tau^{4} A^{2}}\right)\left(I+\frac{\tau^{2}}{6}\right. \\
& \left.\cdot A+\frac{\tau^{4}}{12} A^{2}\right)^{-1},
\end{aligned}
$$

and their conjugate $\widetilde{R}_{5}$,

$$
\begin{aligned}
R_{6}= & \left(I-\frac{1}{3} \tau^{2} A+i \tau A^{1 / 2} \sqrt{I+\frac{1}{72} \tau^{4} A^{2}}\right) \\
& \cdot\left(\frac{\tau^{2}}{2} A+\frac{\tau^{4}}{12} A^{2}-i \tau A^{1 / 2} \sqrt{I+\frac{1}{72} \tau^{4} A^{2}}\right)^{-1},
\end{aligned}
$$

and their conjugate $\widetilde{R}_{6}$,

$R_{7}$

$$
=\left(-2 i \tau A^{1 / 2} \sqrt{I+\frac{1}{72} \tau^{4} A^{2}}\right)\left(I+\frac{\tau^{2}}{6} A+\frac{\tau^{4}}{12} A^{2}\right)^{-1} .
$$


Now we consider the following lemma which was presented before in [14].

Lemma 1. The following estimates hold:

$$
\begin{array}{r}
\|R\|_{H \mapsto H} \leq 1, \\
\|\widetilde{R}\|_{H \mapsto H} \leq 1, \\
\left\|R_{1}\right\|_{H \mapsto H} \leq 1, \\
\left\|\widetilde{R}_{1}\right\|_{H \mapsto H} \leq 1, \\
\left\|A^{1 / 2} R_{2}\right\|_{H \mapsto H} \leq 1, \\
\left\|\tau A^{1 / 2} R_{3}\right\|_{H \mapsto H} \leq 1, \\
\left\|A^{1 / 2} R_{4}\right\|_{H \mapsto H} \leq 1, \\
\left\|A^{-1 / 2} R_{5}\right\|_{H \mapsto H} \leq \tau, \\
\left\|A^{-1 / 2} \widetilde{R}_{5}\right\|_{H \mapsto H} \leq \tau, \\
\left\|\tau A^{1 / 2} R_{6}\right\|_{H \mapsto H} \leq 1, \\
\left\|\tau A^{1 / 2} \widetilde{R}_{6}\right\|_{H \mapsto H} \leq 1, \\
\left\|A^{-1 / 2} \widetilde{R}_{7}\right\|_{H \mapsto H} \leq \tau .
\end{array}
$$

Proof. Using the spectral property of self-adjoint positive definite operators, we write

$$
\begin{aligned}
& \|\left(I-\frac{1}{3} \tau^{2} A \pm i \tau A^{1 / 2} \sqrt{I+\frac{1}{72} \tau^{4} A^{2}}\right) \\
& \cdot\left(I+\frac{1}{6} \tau^{2} A+\frac{1}{12} \tau^{4} A^{2}\right)^{-1} \|_{H \mapsto H} \\
& \quad \leq \operatorname{Sup}_{\delta \leq \lambda<\infty}\left|\frac{1-(1 / 3) \tau^{2} \lambda+i \tau \lambda^{1 / 2} \sqrt{1+(1 / 72) \tau^{4} \lambda^{2}}}{1+(1 / 6) \tau^{2} \lambda+(1 / 12) \tau^{4} \lambda^{2}}\right| .
\end{aligned}
$$

Since

$$
\begin{aligned}
& \frac{\left(1-(1 / 3) \tau^{2} \lambda\right)^{2}+\left(\tau \lambda^{1 / 2} \sqrt{1+(1 / 72) \tau^{4} \lambda^{2}}\right)^{2}}{\left(1+(1 / 6) \tau^{2} \lambda+(1 / 12) \tau^{4} \lambda^{2}\right)^{2}} \\
& =\frac{1+(1 / 3) \tau^{2} \lambda+(1 / 9) \tau^{4} \lambda^{2}+(1 / 72) \tau^{6} \lambda^{3}}{1+(1 / 3) \tau^{2} \lambda+(7 / 36) \tau^{4} \lambda^{2}+(1 / 36) \tau^{6} \lambda^{3}+(1 / 144) \tau^{8} \lambda^{4}} \\
& \leq 1,
\end{aligned}
$$

we have that

$$
\begin{aligned}
& \|R\|_{H \rightarrow H} \leq 1, \\
& \|\widetilde{R}\|_{H \rightarrow H} \leq 1 .
\end{aligned}
$$

In exactly the same manner, one can easily obtain all the other estimates of Lemma 1.
Lemma 2. Suppose that assumption (4) holds. Then, the operator $I-B_{n}^{\tau}$ has an inverse $T_{\tau}=\left(I-B_{n}^{\tau}\right)^{-1}$. From the symmetry and positivity properties of the operator $A$ the following estimate holds:

$$
\left\|T_{\tau}\right\|_{H \mapsto H} \leq M
$$

Proof. Using the definitions of $B_{n}^{\tau}, R, \widetilde{R}$, estimates in (11), and the triangle inequality, we get

$$
\begin{aligned}
& B_{n}^{\tau} \leq \frac{1}{2} \sum_{k=1}^{n}\left|\alpha_{k}\right|\left\{\left(\left\|\widetilde{R}_{1} R^{\left[\lambda_{k} / \tau\right]-1}\right\|_{H \rightarrow H}\right.\right. \\
& \cdot\left\|R_{5}\left(I+\tau^{2} A^{4}\right)^{-1}\right\|_{H \rightarrow H} \\
& \left.+\left\|R_{1} \widetilde{R}^{\left[\lambda_{k} / \tau\right]-1}\right\|_{H \rightarrow H}\left\|\widetilde{R}_{5}\left(I+\tau^{2} A^{4}\right)^{-1}\right\|_{H \rightarrow H}\right) \\
& \cdot\left|\frac{\lambda_{k}}{\tau}-\left[\frac{\lambda_{k}}{\tau}\right]\right|+\frac{7}{6}\left(\left\|\widetilde{R}_{1} R^{\left[\lambda_{k} / \tau\right]-1}\right\|_{H \rightarrow H}\right. \\
& \cdot\left\|R_{5} \tau^{2} A\left(I+\tau^{2} A^{4}\right)^{-1}\right\|_{H \rightarrow H} \\
& \left.+\left\|R_{1} \widetilde{R}^{\left[\lambda_{k} / \tau\right]-1}\right\|_{H \rightarrow H}\left\|\widetilde{R}_{5} \tau^{2} A\left(I+\tau^{2} A^{4}\right)^{-1}\right\|_{H \rightarrow H}\right) \\
& \cdot\left|\frac{\lambda_{k}}{\tau}-\left[\frac{\lambda_{k}}{\tau}\right]\right|^{3}+\left\|\tau^{2} A\left(I+\tau^{2} A^{4}\right)^{-1}\right\|_{H \rightarrow H} \\
& \cdot\left(\left\|\widetilde{R}_{1} R^{\left[\lambda_{k} / \tau\right]}\right\|_{H \rightarrow H}+\left\|R_{1} \widetilde{R}^{\left[\lambda_{k} / \tau\right]}\right\|_{H \rightarrow H}\right) \\
& +\frac{3}{2}\left\|\tau^{2} A\left(I+\tau^{2} A^{4}\right)^{-1}\right\|_{H \rightarrow H} \\
& \left.\cdot\left(\left\|\widetilde{R}_{1} R^{\left[\lambda_{k} / \tau\right]}\right\|_{H \rightarrow H}+\left\|R_{1} \widetilde{R}^{\left[\lambda_{k} / \tau\right]}\right\|_{H \rightarrow H}\right)\left|\frac{\lambda_{k}}{\tau}-\left[\frac{\lambda_{k}}{\tau}\right]\right|^{2}\right\} \\
& -\frac{1}{2} \sum_{k=1}^{n}\left|\beta_{k}\right|\left\|A^{1 / 2} R_{2}\right\|_{H \rightarrow H}\left\{\left\|\left(I+\tau^{2} A^{4}\right)^{-1}\right\|_{H \rightarrow H}\right. \\
& \cdot\left(\left\|\widetilde{R}^{\left[\lambda_{k} / \tau\right]-1}\right\|_{H \rightarrow H}\left\|A^{-1 / 2} \tau^{-1} \widetilde{R}_{5}\right\|_{H \rightarrow H}\right. \\
& \left.+\left\|R^{\left[\lambda_{k} / \tau\right]-1}\right\|_{H \rightarrow H}\left\|A^{-1 / 2} \tau^{-1} R_{5}\right\|_{H \rightarrow H}\right) \\
& +\frac{1}{2}\left(\left\|\widetilde{R}^{\left[\lambda_{k} / \tau\right]-1}\right\|_{H \rightarrow H}\left\|\widetilde{R}_{5} \tau A^{1 / 2}\left(I+\tau^{2} A^{4}\right)^{-1}\right\|_{H \rightarrow H}\right. \\
& \left.+\left\|R^{\left[\lambda_{k} / \tau\right]-1}\right\|_{H \rightarrow H}\left\|R_{5} \tau A^{1 / 2}\left(I+\tau^{2} A^{4}\right)^{-1}\right\|_{H \rightarrow H}\right) \\
& \cdot\left|\frac{\lambda_{k}}{\tau}-\left[\frac{\lambda_{k}}{\tau}\right]\right|^{2}+\left\|A^{1 / 2} \tau\left(I+\tau^{2} A^{4}\right)^{-1}\right\|_{H \rightarrow H} \\
& \cdot\left(\left\|\widetilde{R}^{\left[\lambda_{k} / \tau\right]}\right\|_{H \rightarrow H}+\left\|R^{\left[\lambda_{k} / \tau\right]}\right\|_{H \rightarrow H}\right)\left|\frac{\lambda_{k}}{\tau}-\left[\frac{\lambda_{k}}{\tau}\right]\right| \\
& +\frac{1}{6}\left\|\tau^{3} A^{3 / 2}\left(I+\tau^{2} A^{4}\right)^{-1}\right\|_{H \rightarrow H}
\end{aligned}
$$




$$
\begin{array}{ll}
\left.\cdot\left(\left\|\widetilde{R}^{\left[\lambda_{k} / \tau\right]}\right\|_{H \rightarrow H}+\left\|R^{\left[\lambda_{k} / \tau\right]}\right\|_{H \rightarrow H}\right)\left|\frac{\lambda_{k}}{\tau}-\left[\frac{\lambda_{k}}{\tau}\right]\right|^{3}\right\} & \left.+\frac{1}{2}\left|\frac{\lambda_{k}}{\tau}-\left[\frac{\lambda_{k}}{\tau}\right]\right|^{2}+\frac{1}{6}\left|\frac{\lambda_{k}}{\tau}-\left[\frac{\lambda_{k}}{\tau}\right]\right|^{3}\right\}+\frac{1}{2} \sum_{k=1}^{n}\left|\alpha_{k}\right| \\
-\frac{1}{4} \sum_{k=1}^{n} \sum_{k=1}^{n}\left|\alpha_{k} \beta_{k}\right|\left\|A^{1 / 2} R_{2}\right\|_{H \rightarrow H} & \cdot \sum_{k=1}^{n}\left|\beta_{k}\right|\left\{1+\left|\frac{\lambda_{k}}{\tau}-\left[\frac{\lambda_{k}}{\tau}\right]\right|^{2}+\frac{7}{12}\left|\frac{\lambda_{k}}{\tau}-\left[\frac{\lambda_{k}}{\tau}\right]\right|^{4}\right. \\
\cdot\left\{\left(\left\|\widetilde{R}^{\left[\lambda_{k} / \tau\right]} R^{\left[\lambda_{k} / \tau\right]-1}\right\|_{H \rightarrow H}\right.\right. & \left.+\frac{7}{36}\left|\frac{\lambda_{k}}{\tau}-\left[\frac{\lambda_{k}}{\tau}\right]\right|^{6}\right\} .
\end{array}
$$$$
\cdot\left\|\left(\widetilde{R}_{1}-R_{1}\right) R_{5} \tau^{-1} A^{-1 / 2}\left(I+\tau^{2} A^{4}\right)^{-1}\right\|_{H \rightarrow H}
$$$$
+\left\|R^{\left[\lambda_{k} / \tau\right]} \widetilde{R}^{\left[\lambda_{k} / \tau\right]-1}\right\|_{H \rightarrow H}
$$$$
\text { . } \left.\left\|\left(\widetilde{R}_{1}-R_{1}\right) \widetilde{R}_{5} \tau^{-1} A^{-1 / 2}\left(I+\tau^{2} A^{4}\right)^{-1}\right\|_{H \rightarrow H}\right)
$$$$
\cdot\left(\left\|\widetilde{R}^{\left[\lambda_{k} / \tau\right]} R^{\left[\lambda_{k} / \tau\right]-1}\right\|_{H \rightarrow H}\right.
$$$$
\text { . }\left\|\left(\widetilde{R}_{1}-R_{1}\right) R_{5} \tau^{-1} A^{1 / 2}\left(I+\tau^{2} A^{4}\right)^{-1}\right\|_{H \rightarrow H}
$$$$
+\left\|R^{\left[\lambda_{k} / \tau\right]} \widetilde{R}^{\left[\lambda_{k} / \tau\right]-1}\right\|_{H \rightarrow H}
$$$$
\left.\cdot\left\|\left(\widetilde{R}_{1}-R_{1}\right) \widetilde{R}_{5} \tau A^{1 / 2}\left(I+\tau^{2} A^{4}\right)^{-1}\right\|_{H \rightarrow H}\right)
$$$$
\cdot\left|\frac{\lambda_{k}}{\tau}-\left[\frac{\lambda_{k}}{\tau}\right]\right|^{2}+\frac{7}{12}
$$$$
\cdot\left(\left\|\widetilde{R}^{\left[\lambda_{k} / \tau\right]} R^{\left[\lambda_{k} / \tau\right]-1}\right\|_{H \rightarrow H}\right.
$$$$
\cdot\left\|\left(\widetilde{R}_{1}-R_{1}\right) R_{5} A^{3 / 2} \tau^{3}\left(I+\tau^{2} A^{4}\right)^{-1}\right\|_{H \rightarrow H}
$$$$
+\left\|R^{\left[\lambda_{k} / \tau\right]} \widetilde{R}^{\left[\lambda_{k} / \tau\right]-1}\right\|_{H \rightarrow H}
$$$$
\left.\cdot\left\|\left(\widetilde{R}_{1}-R_{1}\right) \widetilde{R}_{5} \tau^{3} A^{3 / 2}\left(I+\tau^{2} A^{4}\right)^{-1}\right\|_{H \rightarrow H}\right)
$$$$
\cdot\left|\frac{\lambda_{k}}{\tau}-\left[\frac{\lambda_{k}}{\tau}\right]\right|^{4}+\frac{7}{36}
$$$$
\cdot\left(\left\|\widetilde{R}^{\left[\lambda_{k} / \tau\right]} R^{\left[\lambda_{k} / \tau\right]-1}\right\|_{H \rightarrow H}\right.
$$$$
\cdot\left\|\left(\widetilde{R}_{1}-R_{1}\right) R_{5} \tau^{5} A^{5 / 2}\left(I+\tau^{2} A^{4}\right)^{-1}\right\|_{H \rightarrow H}
$$$$
+\left\|R^{\left[\lambda_{k} / \tau\right]} \widetilde{R}^{\left[\lambda_{k} / \tau\right]-1}\right\|_{H \rightarrow H}
$$$$
\left.\cdot\left\|\left(\widetilde{R}_{1}-R_{1}\right) \widetilde{R}_{5} \tau^{5} A^{5 / 2}\left(I+\tau^{2} A^{4}\right)^{-1}\right\|_{H \rightarrow H}\right)
$$$$
\left.\left.\cdot\left|\frac{\lambda_{k}}{\tau}-\left[\frac{\lambda_{k}}{\tau}\right]\right|\right|^{6}\right\} \leq q \text {, }
$$

where

$$
\begin{aligned}
q= & \sum_{k=1}^{n}\left|\alpha_{k}\right|\left\{1+\left|\frac{\lambda_{k}}{\tau}-\left[\frac{\lambda_{k}}{\tau}\right]\right|+\frac{3}{2}\left|\frac{\lambda_{k}}{\tau}-\left[\frac{\lambda_{k}}{\tau}\right]\right|^{2}\right. \\
& \left.+\frac{7}{6}\left|\frac{\lambda_{k}}{\tau}-\left[\frac{\lambda_{k}}{\tau}\right]\right|^{3}\right\}+\sum_{k=1}^{n}\left|\beta_{k}\right|\left\{1+\left|\frac{\lambda_{k}}{\tau}-\left[\frac{\lambda_{k}}{\tau}\right]\right|\right.
\end{aligned}
$$

Since $q<1$, the operator $I-B_{n}^{\tau}$ has a bounded inverse and

$$
\left\|\left(I-B_{n}^{\tau}\right)^{-1}\right\|_{H \rightarrow H} \leq \frac{1}{1-q} .
$$

Lemma 2 is proved.

Note that the stability estimates in the sequel are obtained by the method presented in the proofs of Lemmas 1 and 2 to obtain stability estimates. For clarity and avoiding long equations the proofs of estimates are not put.

Now, applying the following formula (see [14])

$$
\begin{aligned}
u_{0}= & \mu, \\
u_{1}= & \left(I+\frac{\tau^{2}}{12} A+\frac{\tau^{4}}{144} A^{2}\right)^{-1} \\
& \cdot\left(\left(I-\frac{5}{12} \tau^{2} A+\frac{\tau^{4}}{144} A^{2}\right) \mu\right. \\
& \left.+\tau\left(I-\frac{\tau^{2}}{12} A\right) \omega+\tau^{2} f_{1,1}\right), \\
u_{k} & =\frac{1}{2}\left[\widetilde{R}_{1} R^{k}-R_{1} \widetilde{R}^{k}\right] \mu+\frac{1}{2}\left[\widetilde{R}^{k}-R^{k}\right] R_{2} \omega+\frac{1}{2}\left[\widetilde{R}^{k}\right. \\
& \left.-R^{k}\right] R_{3} \tau^{2} f_{1,1}+\frac{1}{2} R_{4} \sum_{s=1}^{k-1}\left[\widetilde{R}^{k-s}-R^{k-s}\right] f_{s} \tau^{2},
\end{aligned}
$$

and the multipoint nonlocal boundary conditions in (2), we obtain

$$
\begin{aligned}
\mu= & T_{\tau}\left(I+i \tau^{2} A^{4}\right)^{-1}\left\{\left(I-\frac{1}{2} R_{2}\right.\right. \\
& \cdot \sum_{k=1}^{n} \beta_{k}\left\{\tau^{-1}\left(\widetilde{R}^{\left[\lambda_{k} / \tau\right]-1} \widetilde{R}_{5}+R^{\left[\lambda_{k} / \tau\right]-1} R_{5}\right)\right. \\
& +\frac{1}{2} \tau A\left(\widetilde{R}^{\left[\lambda_{k} / \tau\right]-1} \widetilde{R}_{5}+R^{\left[\lambda_{k} / \tau\right]-1} R_{5}\right) \\
& \cdot\left|\frac{\lambda_{k}}{\tau}-\left[\frac{\lambda_{k}}{\tau}\right]\right|^{2}-A \tau\left(\widetilde{R}^{\left[\lambda_{k} / \tau\right]}+R^{\left[\lambda_{k} / \tau\right]}\right)\left|\frac{\lambda_{k}}{\tau}-\left[\frac{\lambda_{k}}{\tau}\right]\right| \\
& \left.\left.+\frac{1}{6} \tau^{3} A^{2}\left[\widetilde{R}^{\left[\lambda_{k} / \tau\right]}+R^{\left[\lambda_{k} / \tau\right]}\right]\left|\frac{\lambda_{k}}{\tau}-\left[\frac{\lambda_{k}}{\tau}\right]\right|^{3}\right\}\right)
\end{aligned}
$$




$$
\begin{aligned}
& \cdot\left(\sum _ { k = 1 } ^ { n } \frac { 1 } { 2 } \alpha _ { k } R _ { 3 } \tau ^ { 2 } f _ { 1 , 1 } \left\{\left(\widetilde{R}^{\left[\lambda_{k} / \tau\right]-1} \widetilde{R}_{5}+R^{\left[\lambda_{k} / \tau\right]-1} R_{5}\right)\right.\right. \\
& \cdot\left|\frac{\lambda_{k}}{\tau}-\left[\frac{\lambda_{k}}{\tau}\right]\right|-\frac{7}{6} A \tau^{2}\left(R^{\left[\lambda_{k} / \tau\right]-1} R_{5}+\widetilde{R}^{\left[\lambda_{k} / \tau\right]-1} \widetilde{R}_{5}\right) \\
& \cdot\left|\frac{\lambda_{k}}{\tau}-\left[\frac{\lambda_{k}}{\tau}\right]\right|^{3}+\left[\widetilde{R}^{\left[\lambda_{k} / \tau\right]}+R^{\left[\lambda_{k} / \tau\right]}\right] \\
& \left.-\frac{3}{2} \tau^{2} A\left[\widetilde{R}^{\left[\lambda_{k} / \tau\right]}+R^{\left[\lambda_{k} / \tau\right]}\right]\left|\frac{\lambda_{k}}{\tau}-\left[\frac{\lambda_{k}}{\tau}\right]\right|^{2}\right\} \\
& +\frac{1}{2} R_{4} \sum_{k=1}^{n} \alpha_{k} \sum_{s=1}^{\left[\lambda_{k} / \tau\right]-2} f_{s} \tau^{2}\left\{\left(\widetilde{R}^{\left[\lambda_{k} / \tau\right]-s-1} \widetilde{R}_{5}+R^{\left[\lambda_{k} / \tau\right]-s-1} R_{5}\right)\right. \\
& \cdot\left|\frac{\lambda_{k}}{\tau}-\left[\frac{\lambda_{k}}{\tau}\right]\right| \\
& \left.-\frac{7}{6} A \tau^{2}\left(\widetilde{R}^{\left[\lambda_{k} / \tau\right]-s-1} \widetilde{R}_{5}+R^{\left[\lambda_{k} / \tau\right]-s-1} R_{5}\right)\left|\frac{\lambda_{k}}{\tau}-\left[\frac{\lambda_{k}}{\tau}\right]\right|^{3}\right\} \\
& +\frac{1}{2} R_{4} \sum_{k=1}^{n} \alpha_{k} \sum_{s=1}^{\left[\lambda_{k} / \tau\right]-1} f_{s} \tau^{2}\left(\widetilde{R}^{\left[\lambda_{k} / \tau\right]-s}+R^{\left[\lambda_{k} / \tau\right]-s}\right) \\
& \left(1-\frac{3}{2} A \tau^{2}\left|\frac{\lambda_{k}}{\tau}-\left[\frac{\lambda_{k}}{\tau}\right]\right|^{2}\right) \frac{1}{2} R_{4} \\
& \cdot \sum_{k=1}^{n} \alpha_{k} R_{7}\left\{\left|\frac{\lambda_{k}}{\tau}-\left[\frac{\lambda_{k}}{\tau}\right]\right|-\frac{7}{6} A \tau^{2}\left|\frac{\lambda_{k}}{\tau}-\left[\frac{\lambda_{k}}{\tau}\right]\right|^{3}\right\} \\
& \cdot f_{\left[\lambda_{k} / \tau\right]-1} \tau^{2}+\sum_{k=1}^{n} \alpha_{k}\left\{\frac{3}{2}\left|\frac{\lambda_{k}}{\tau}-\left[\frac{\lambda_{k}}{\tau}\right]\right|^{2} f_{\left[\lambda_{k} / \tau\right]} \tau^{2}\right. \\
& \left.\left.+\frac{7}{6}\left|\frac{\lambda_{k}}{\tau}-\left[\frac{\lambda_{k}}{\tau}\right]\right|^{3} f_{\left[\lambda_{k} / \tau\right]}^{\prime} \tau^{3}\right\}+\varphi\right) \\
& -\left(-\frac{1}{2} R_{2} \sum_{k=1}^{n} \alpha_{k}\left\{\left(\widetilde{R}_{1} R^{\left[\lambda_{k} / \tau\right]-1} R_{5}+R_{1} \widetilde{R}^{\left[\lambda_{k} / \tau\right]-1} \widetilde{R}_{5}\right)\right.\right. \\
& \cdot\left|\frac{\lambda_{k}}{\tau}-\left[\frac{\lambda_{k}}{\tau}\right]\right|-\frac{7}{6} \tau^{2} A\left(\widetilde{R}_{1} R^{\left[\lambda_{k} / \tau\right]-1} R_{5}+R_{1} \widetilde{R}^{\left[\lambda_{k} / \tau\right]-1} \widetilde{R}_{5}\right) \\
& \cdot\left|\frac{\lambda_{k}}{\tau}-\left[\frac{\lambda_{k}}{\tau}\right]\right|^{3}+\left(\widetilde{R}_{1} R^{\left[\lambda_{k} / \tau\right]}+R_{1} \widetilde{R}^{\left[\lambda_{k} / \tau\right]}\right) \\
& \left.\left.-\frac{3}{2} A \tau^{2}\left(\widetilde{R}_{1} R^{\left[\lambda_{k} / \tau\right]}+R_{1} \widetilde{R}^{\left[\lambda_{k} / \tau\right]}\right)\left|\frac{\lambda_{k}}{\tau}-\left[\frac{\lambda_{k}}{\tau}\right]^{2}\right|\right\}\right) \\
& \cdot\left(R _ { 3 } \sum _ { k = 1 } ^ { n } \frac { 1 } { 2 } \beta _ { k } f _ { 1 , 1 } \tau ^ { 2 } \left\{\tau^{-1}\left(\widetilde{R}^{\left[\lambda_{k} / \tau\right]-1} \widetilde{R}_{5}+R^{\left[\lambda_{k} / \tau\right]-1} R_{5}\right)\right.\right. \\
& -\frac{1}{2} \tau A\left(\widetilde{R}^{\left[\lambda_{k} / \tau\right]-1} \widetilde{R}_{5}+R^{\left[\lambda_{k} / \tau\right]-1} R_{5}\right)\left|\frac{\lambda_{k}}{\tau}-\left[\frac{\lambda_{k}}{\tau}\right]\right|^{2} \\
& -A \tau\left(\widetilde{R}^{\left[\lambda_{k} / \tau\right]}+R^{\left[\lambda_{k} / \tau\right]}\right)\left|\frac{\lambda_{k}}{\tau}-\left[\frac{\lambda_{k}}{\tau}\right]\right| \\
& \left.+\frac{1}{6} A^{2} \tau^{3}\left(\widetilde{R}^{\left[\lambda_{k} / \tau\right]}+R^{\left[\lambda_{k} / \tau\right]}\right)\left|\frac{\lambda_{k}}{\tau}-\left[\frac{\lambda_{k}}{\tau}\right]\right|^{3}\right\} \\
& +\frac{1}{2} \sum_{k=1}^{n} \sum_{s=1}^{\left[\lambda_{k} / \tau\right]-2} R_{4} \beta_{k}\left(\widetilde{R}^{\left[\lambda_{k} / \tau\right]-s-1} \widetilde{R}_{5}+R^{\left[\lambda_{k} / \tau\right]-s-1} R_{5}\right)
\end{aligned}
$$

$$
\begin{aligned}
& \cdot\left(\frac{1}{\tau}-\frac{1}{2} A \tau\left|\frac{\lambda_{k}}{\tau}-\left[\frac{\lambda_{k}}{\tau}\right]\right|^{2}\right) f_{s} \tau^{2} \\
& +\frac{1}{2} \sum_{k=1}^{n} \sum_{s=1}^{\left[\lambda_{k} / \tau\right]-1} R_{4} \beta_{k}\left(\widetilde{R}^{\left[\lambda_{k} / \tau\right]-s}+R^{\left[\lambda_{k} / \tau\right]-s}\right) \\
& \cdot\left(-A \tau\left|\frac{\lambda_{k}}{\tau}-\left[\frac{\lambda_{k}}{\tau}\right]\right|+\frac{1}{6} A \tau^{2}\left|\frac{\lambda_{k}}{\tau}-\left[\frac{\lambda_{k}}{\tau}\right]\right|^{3}\right) f_{s} \tau^{2} \\
& +\frac{1}{2} \sum_{k=1}^{n} R_{4} \beta_{k} R_{7}\left(\frac{1}{\tau}-\frac{1}{2} A \tau\left|\frac{\lambda_{k}}{\tau}-\left[\frac{\lambda_{k}}{\tau}\right]\right|^{2}\right) f_{\left[\lambda_{k} / \tau\right]-1} \tau^{2} \\
& +\sum_{k=1}^{n} \beta_{k}\left\{-\frac{1}{6} A \tau^{3}\left|\frac{\lambda_{k}}{\tau}-\left[\frac{\lambda_{k}}{\tau}\right]\right|^{3} f_{\left[\lambda_{k} / \tau\right]}\right. \\
& +\left|\frac{\lambda_{k}}{\tau}-\left[\frac{\lambda_{k}}{\tau}\right]\right| f_{\left[\lambda_{k} / \tau\right]} \tau+\frac{1}{2}\left|\frac{\lambda_{k}}{\tau}-\left[\frac{\lambda_{k}}{\tau}\right]\right|^{2} f_{\left[\lambda_{k} / \tau\right]}^{\prime} \tau^{2} \\
& \left.\left.\left.+\frac{1}{6} \tau^{3}\left|\frac{\lambda_{k}}{\tau}-\left[\frac{\lambda_{k}}{\tau}\right]\right|^{3} f_{\left[\lambda_{k} / \tau\right]}^{\prime \prime}\right\}+\psi\right)\right\} \text {, } \\
& \omega=T_{\tau}\left(I+i \tau^{2} A^{4}\right)^{-1} \\
& \cdot\left\{\left(1-\frac{1}{2} \sum_{k=1}^{n} a_{k}\left\{\left(\widetilde{R}_{1} R^{\left[\lambda_{k} / \tau\right]-1} R_{5}+R_{1} \widetilde{R}^{\left[\lambda_{k} / \tau\right]-1} \widetilde{R}_{5}\right)\right.\right.\right. \\
& \cdot\left|\frac{\lambda_{k}}{\tau}-\left[\frac{\lambda_{k}}{\tau}\right]\right|-\frac{7}{6} A \tau^{2}\left(\widetilde{R}_{1} R^{\left[\lambda_{k} / \tau\right]-1} R_{5}+R_{1} \widetilde{R}^{\left[\lambda_{k} / \tau\right]-1} \widetilde{R}_{5}\right) \\
& \cdot\left|\frac{\lambda_{k}}{\tau}-\left[\frac{\lambda_{k}}{\tau}\right]\right|^{3}+\left(\widetilde{R}_{1} R^{\left[\lambda_{k} / \tau\right]}+R_{1} \widetilde{R}^{\left[\lambda_{k} / \tau\right]}\right) \\
& \left.\left.\cdot\left(1-\frac{3}{2} A \tau^{2}\left|\frac{\lambda_{k}}{\tau}-\left[\frac{\lambda_{k}}{\tau}\right]\right|^{2}\right)\right\}\right) \\
& \cdot\left(R _ { 3 } \sum _ { k = 1 } ^ { n } \frac { 1 } { 2 } \beta _ { k } f _ { 1 , 1 } \tau ^ { 2 } \left\{\tau^{-1}\left(\widetilde{R}^{\left[\lambda_{k} / \tau\right]-1} \widetilde{R}_{5}+R^{\left[\lambda_{k} / \tau\right]-1} R_{5}\right)\right.\right. \\
& -\frac{1}{2} \tau A\left(\widetilde{R}^{\left[\lambda_{k} / \tau\right]-1} \widetilde{R}_{5}+R^{\left[\lambda_{k} / \tau\right]-1} R_{5}\right) \\
& \cdot\left|\frac{\lambda_{k}}{\tau}-\left[\frac{\lambda_{k}}{\tau}\right]\right|^{2}-A \tau\left(\widetilde{R}^{\left[\lambda_{k} / \tau\right]}+R^{\left[\lambda_{k} / \tau\right]}\right)\left|\frac{\lambda_{k}}{\tau}-\left[\frac{\lambda_{k}}{\tau}\right]\right| \\
& \left.+\frac{1}{6} A^{2} \tau^{3}\left(\widetilde{R}^{\left[\lambda_{k} / \tau\right]}+R^{\left[\lambda_{k} / \tau\right]}\right)\left|\frac{\lambda_{k}}{\tau}-\left[\frac{\lambda_{k}}{\tau}\right]\right|^{3}\right\} \\
& +\frac{1}{2} \sum_{k=1}^{n} \sum_{s=1}^{\left[\lambda_{k} / \tau\right]-2} R_{4} \beta_{k} f_{s} \tau^{2}\left(\widetilde{R}^{\left[\lambda_{k} / \tau\right]-s-1} \widetilde{R}_{5}+R^{\left[\lambda_{k} / \tau\right]-s-1} R_{5}\right) \\
& \cdot\left(\frac{1}{\tau}-\frac{1}{2} A \tau\left|\frac{\lambda_{k}}{\tau}-\left[\frac{\lambda_{k}}{\tau}\right]\right|^{2}\right) \\
& +\frac{1}{2} \sum_{k=1}^{n} \sum_{s=1}^{\left[\lambda_{k} / \tau\right]-1} R_{4} \beta_{k}\left(\widetilde{R}^{\left[\lambda_{k} / \tau\right]-s}+R^{\left[\lambda_{k} / \tau\right]-s}\right) \\
& \cdot\left(-A \tau\left|\frac{\lambda_{k}}{\tau}-\left[\frac{\lambda_{k}}{\tau}\right]\right|+\frac{1}{6} A \tau^{2}\left|\frac{\lambda_{k}}{\tau}-\left[\frac{\lambda_{k}}{\tau}\right]\right|^{3}\right) f_{s} \tau^{2} \\
& +\frac{1}{2} \sum_{k=1}^{n} R_{4} \beta_{k} R_{7}\left(\frac{1}{\tau}-\frac{1}{2} A \tau\left|\frac{\lambda_{k}}{\tau}-\left[\frac{\lambda_{k}}{\tau}\right]\right|^{2}\right) f_{\left[\lambda_{k} / \tau\right]-1} \tau^{2}
\end{aligned}
$$




$$
\begin{aligned}
& +\sum_{k=1}^{n} \beta_{k}\left\{-\frac{1}{6} A \tau^{3}\left|\frac{\lambda_{k}}{\tau}-\left[\frac{\lambda_{k}}{\tau}\right]\right|^{3} f_{\left[\lambda_{k} / \tau\right]}\right. \\
& +\left|\frac{\lambda_{k}}{\tau}-\left[\frac{\lambda_{k}}{\tau}\right]\right| f_{\left[\lambda_{k} / \tau\right]} \tau \\
& +\frac{1}{2}\left|\frac{\lambda_{k}}{\tau}-\left[\frac{\lambda_{k}}{\tau}\right]\right|^{2} f_{\left[\lambda_{k} / \tau\right]}^{\prime} \tau^{2} \\
& \left.\left.\left.+\frac{1}{6} \tau^{3}\left|\frac{\lambda_{k}}{\tau}-\left[\frac{\lambda_{k}}{\tau}\right]\right|^{3} f_{\left[\lambda_{k} / \tau\right]}^{\prime \prime}\right\}+\psi\right)\right\} \\
& -\left(-\frac{1}{2} \sum_{k=1}^{n} \beta_{k}\left\{\frac{1}{\tau}\left(\widetilde{R}_{1} R^{\left[\lambda_{k} / \tau\right]-1} R_{5}+R_{1} \widetilde{R}^{\left[\lambda_{k} / \tau\right]-1} \widetilde{R}_{5}\right)\right.\right. \\
& +\frac{1}{2} A \tau\left(\widetilde{R}_{1} R^{\left[\lambda_{k} / \tau\right]-1} R_{5}+R_{1} \widetilde{R}^{\left[\lambda_{k} / \tau\right]-1} \widetilde{R}_{5}\right)\left|\frac{\lambda_{k}}{\tau}-\left[\frac{\lambda_{k}}{\tau}\right]\right|^{2} \\
& -A \tau\left(\widetilde{R}_{1} R^{\left[\lambda_{k} / \tau\right]}+R_{1} \widetilde{R}^{\left[\lambda_{k} / \tau\right]}\right)\left|\frac{\lambda_{k}}{\tau}-\left[\frac{\lambda_{k}}{\tau}\right]\right| \\
& \left.\left.+\frac{1}{6} A^{2} \tau^{3}\left(\widetilde{R}_{1} R^{\left[\lambda_{k} / \tau\right]}+R_{1} \widetilde{R}^{\left[\lambda_{k} / \tau\right]}\right)\left|\frac{\lambda_{k}}{\tau}-\left[\frac{\lambda_{k}}{\tau}\right]\right|^{3}\right\}\right) \\
& \cdot\left(\sum _ { k = 1 } ^ { n } \frac { 1 } { 2 } \alpha _ { k } R _ { 3 } \tau ^ { 2 } f _ { 1 , 1 } \left\{\left(\widetilde{R}^{\left[\lambda_{k} / \tau\right]-1} \widetilde{R}_{5}+R^{\left[\lambda_{k} / \tau\right]-1} R_{5}\right)\right.\right. \\
& \cdot\left|\frac{\lambda_{k}}{\tau}-\left[\frac{\lambda_{k}}{\tau}\right]\right|+\frac{7}{6} A \tau^{2}\left(R^{\left[\lambda_{k} / \tau\right]-1} R_{5}+\widetilde{R}^{\left[\lambda_{k} / \tau\right]-1} \widetilde{R}_{5}\right) \\
& \cdot\left|\frac{\lambda_{k}}{\tau}-\left[\frac{\lambda_{k}}{\tau}\right]\right|^{3}+\left[\widetilde{R}^{\left[\lambda_{k} / \tau\right]}+R^{\left[\lambda_{k} / \tau\right]}\right] \\
& \left.-\frac{3}{2} \tau^{2} A\left[\widetilde{R}^{\left[\lambda_{k} / \tau\right]}+R^{\left[\lambda_{k} / \tau\right]}\right]\left|\frac{\lambda_{k}}{\tau}-\left[\frac{\lambda_{k}}{\tau}\right]\right|^{2}\right\} \\
& +\frac{1}{2} R_{4} \sum_{k=1}^{n} \alpha_{k} \sum_{s=1}^{\left[\lambda_{k} / \tau\right]-2} f_{s} \tau^{2}\left\{\left(\widetilde{R}^{\left[\lambda_{k} / \tau\right]-s-1} \widetilde{R}_{5}+R^{\left[\lambda_{k} / \tau\right]-s-1} R_{5}\right)\right. \\
& \cdot\left|\frac{\lambda_{k}}{\tau}-\left[\frac{\lambda_{k}}{\tau}\right]\right|+\frac{7}{6} A \tau^{2}\left(\widetilde{R}^{\left[\lambda_{k} / \tau\right]-s-1} \widetilde{R}_{5}+R^{\left[\lambda_{k} / \tau\right]-s-1} R_{5}\right) \\
& \left.\cdot\left|\frac{\lambda_{k}}{\tau}-\left[\frac{\lambda_{k}}{\tau}\right]\right|^{3}\right\}+\frac{1}{2} R_{4} \\
& \cdot \sum_{k=1}^{n} \alpha_{k} \sum_{s=1}^{\left[\lambda_{k} / \tau\right]-1} f_{s} \tau^{2}\left(\widetilde{R}^{\left[\lambda_{k} / \tau\right]-s}+R^{\left[\lambda_{k} / \tau\right]-s}\right) \\
& \left(1-\frac{3}{2} A \tau^{2}\left|\frac{\lambda_{k}}{\tau}-\left[\frac{\lambda_{k}}{\tau}\right]\right|^{2}\right) \frac{1}{2} R_{4} \\
& \cdot \sum_{k=1}^{n} \alpha_{k} R_{7}\left\{\left|\frac{\lambda_{k}}{\tau}-\left[\frac{\lambda_{k}}{\tau}\right]\right|-\frac{7}{6} A \tau^{2}\left|\frac{\lambda_{k}}{\tau}-\left[\frac{\lambda_{k}}{\tau}\right]\right|^{3}\right\} \\
& \cdot f_{\left[\lambda_{k} / \tau\right]-1} \tau^{2}+\sum_{k=1}^{n} \alpha_{k}\left\{\frac{3}{2}\left|\frac{\lambda_{k}}{\tau}-\left[\frac{\lambda_{k}}{\tau}\right]\right|^{2} f_{\left[\lambda_{k} / \tau\right]} \tau^{2}\right. \\
& \left.\left.+\frac{7}{6}\left|\frac{\lambda_{k}}{\tau}-\left[\frac{\lambda_{k}}{\tau}\right]\right|^{3} f_{\left[\lambda_{k} / \tau\right]}^{\prime} \tau^{3}\right\}+\varphi\right) \text {. }
\end{aligned}
$$

Formulas (19) and (20) give a solution of problem (2).
Theorem 3. Suppose that assumptions (4) and (11) hold and $\varphi \in D\left(A^{3 / 2}\right)$ and $\psi \in D\left(A^{1 / 2}\right)$. Then, for the solution of difference scheme (2), the following stability estimates hold:

$$
\begin{aligned}
& \max _{1 \leq k \leq N}\left\|u_{k}\right\|_{H} \leq M\left\{\sum_{s=1}^{k-1}\left\|A^{-1 / 2} f_{s}\right\|_{H} \tau+\|\varphi\|_{H}\right. \\
& \left.+\left\|A^{-1 / 2} \psi\right\|_{H}+\tau\left\|A^{-1 / 2} f_{1,1}\right\|_{H}\right\}, \\
& \max _{1 \leq k \leq N}\left\|A^{1 / 2} u_{k}\right\|_{H} \leq M\left\{\sum_{s=1}^{k-1}\left\|f_{s}\right\|_{H} \tau+\left\|A^{1 / 2} \varphi\right\|_{H}\right. \\
& \left.+\|\psi\|_{H}+\tau\left\|f_{1,1}\right\|_{H}\right\}, \\
& \max _{1 \leq k \leq N}\left\|A u_{k}\right\|_{H} \leq M\left\{\sum_{s=2}^{N-1}\left\|f_{s}-f_{s-1}\right\|_{H}+\left\|f_{1}\right\|_{H}\right. \\
& \left.+\|A \varphi\|_{H}+\left\|A^{1 / 2} \psi\right\|_{H}+\tau\left\|A^{1 / 2} f_{1,1}\right\|_{H}\right\},
\end{aligned}
$$

where $M$ does not depend on $\tau, \varphi, \psi, f_{1,1}$ and $f_{s}, 1 \leq s \leq N-1$.

Proof. Using formulas (20), estimates (11) and (15), and the method of Lemmas 1 and 2, we get

$$
\begin{aligned}
& \|\mu\|_{H} \leq M\left\{\sum_{s=1}^{k-1}\left\|A^{-1 / 2} f_{s}\right\|_{H} \tau+\|\varphi\|_{H}+\left\|A^{-1 / 2} \psi\right\|_{H}\right. \\
& \left.+\tau\left\|A^{-1 / 2} f_{1,1}\right\|_{H}\right\}, \\
& \left\|A^{-1 / 2} \omega\right\|_{H} \leq M\left\{\sum_{s=1}^{k-1}\left\|A^{-1 / 2} f_{s}\right\|_{H} \tau+\|\varphi\|_{H}\right. \\
& \left.+\left\|A^{-1 / 2} \psi\right\|_{H}+\tau\left\|A^{-1 / 2} f_{1,1}\right\|_{H}\right\} .
\end{aligned}
$$

Applying $A^{1 / 2}$ to formulas (20), we obtain

$$
\begin{aligned}
& \left\|A^{1 / 2} \mu\right\|_{H} \\
& \leq M\left\{\sum_{s=1}^{k-1}\left\|f_{s}\right\|_{H} \tau+\left\|A^{1 / 2} \varphi\right\|_{H}+\|\psi\|_{H}+\tau\left\|f_{1,1}\right\|_{H}\right\}, \\
& \|\omega\|_{H} \\
& \leq M\left\{\sum_{s=1}^{k-1}\left\|f_{s}\right\|_{H} \tau+\left\|A^{1 / 2} \varphi\right\|_{H}+\|\psi\|_{H}+\tau\left\|f_{1,1}\right\|_{H}\right\} .
\end{aligned}
$$


Now, applying Abel's formula to (20), we get

$$
\begin{aligned}
& \mu=T_{\tau}\left(I+i \tau^{2} A^{4}\right)^{-1} \\
& \left\{\left(I-\frac{1}{2} R_{2} \sum_{k=1}^{n} \beta_{k}\left\{\tau^{-1}\left(\widetilde{R}^{\left[\lambda_{k} / \tau\right]-1} \widetilde{R}_{5}+R^{\left[\lambda_{k} / \tau\right]-1} R_{5}\right)\right.\right.\right. \\
& +\frac{1}{2} \tau A\left(\widetilde{R}^{\left[\lambda_{k} / \tau\right]-1} \widetilde{R}_{5}+R^{\left[\lambda_{k} / \tau\right]-1} R_{5}\right)\left|\frac{\lambda_{k}}{\tau}-\left[\frac{\lambda_{k}}{\tau}\right]\right|^{2} \\
& -A \tau\left(\widetilde{R}^{\left[\lambda_{k} / \tau\right]}+R^{\left[\lambda_{k} / \tau\right]}\right)\left|\frac{\lambda_{k}}{\tau}-\left[\frac{\lambda_{k}}{\tau}\right]\right| \\
& \left.\left.+\frac{1}{6} \tau^{3} A^{2}\left[\widetilde{R}^{\left[\lambda_{k} / \tau\right]}+R^{\left[\lambda_{k} / \tau\right]}\right]\left|\frac{\lambda_{k}}{\tau}-\left[\frac{\lambda_{k}}{\tau}\right]\right|^{3}\right\}\right) \\
& \cdot\left(\sum_{k=1}^{n} \frac{1}{2} \alpha_{k} R_{3} \tau^{2} f_{1,1}\right. \\
& \left\{\left(\widetilde{R}^{\left[\lambda_{k} / \tau\right]-1} \widetilde{R}_{5}+R^{\left[\lambda_{k} / \tau\right]-1} R_{5}\right)\left|\frac{\lambda_{k}}{\tau}-\left[\frac{\lambda_{k}}{\tau}\right]\right|\right. \\
& -\frac{7}{6} A \tau^{2}\left(R^{\left[\lambda_{k} / \tau\right]-1} R_{5}+\widetilde{R}^{\left[\lambda_{k} / \tau\right]-1} \widetilde{R}_{5}\right)\left|\frac{\lambda_{k}}{\tau}-\left[\frac{\lambda_{k}}{\tau}\right]\right|^{3} \\
& +\left[\widetilde{R}^{\left[\lambda_{k} / \tau\right]}+R^{\left[\lambda_{k} / \tau\right]}\right] \\
& \left.-\frac{3}{2} \tau^{2} A\left[\widetilde{R}^{\left[\lambda_{k} / \tau\right]}+R^{\left[\lambda_{k} / \tau\right]}\right]\left|\frac{\lambda_{k}}{\tau}-\left[\frac{\lambda_{k}}{\tau}\right]\right|^{2}\right\} \\
& +\frac{1}{2} R_{4} \tau^{2} \sum_{k=1}^{n} \alpha_{k} \\
& \cdot\left[\sum _ { s = 2 } ^ { [ \lambda _ { k } / \tau ] - 2 } \left(\left(R_{5} R_{6} R^{\left[\lambda_{k} / \tau\right]-s-1}-\widetilde{R}_{5} \widetilde{R}_{6} \widetilde{R}^{\left[\lambda_{k} / \tau\right]-s-1}\right)\right.\right. \\
& \cdot\left|\frac{\lambda_{k}}{\tau}-\left[\frac{\lambda_{k}}{\tau}\right]\right| \\
& -\frac{7}{6} A \tau^{2}\left(R_{5} R_{6} R^{\left[\lambda_{k} / \tau\right]-s-1}-\widetilde{R}_{5} \widetilde{R}_{6} \widetilde{R}^{\left[\lambda_{k} / \tau\right]-s-1}\right) \\
& \left.\cdot\left|\frac{\lambda_{k}}{\tau}-\left[\frac{\lambda_{k}}{\tau}\right]\right|^{3}\right)\left(f_{s}-f_{s-1}\right) \\
& +\left(\left(\widetilde{R}_{5} \widetilde{R}_{6}-R_{5} R_{6}\right)\left|\frac{\lambda_{k}}{\tau}-\left[\frac{\lambda_{k}}{\tau}\right]\right|\right. \\
& \left.-\frac{7}{6} A \tau^{2}\left(\widetilde{R}_{5} \widetilde{R}_{6}-R_{5} R_{6}\right)\left|\frac{\lambda_{k}}{\tau}-\left[\frac{\lambda_{k}}{\tau}\right]\right|^{3}\right) f_{\left[\lambda_{k} / \tau\right]-2} \\
& -\left(\left(\widetilde{R}_{5} \widetilde{R}_{6} \widetilde{R}^{\left[\lambda_{k} / \tau\right]-1}-R_{5} R_{6} R^{\left[\lambda_{k} / \tau\right]-1}\right)\left|\frac{\lambda_{k}}{\tau}-\left[\frac{\lambda_{k}}{\tau}\right]\right|\right. \\
& +\frac{7}{6} A \tau^{2}\left(\widetilde{R}_{5} \widetilde{R}_{6} \widetilde{R}^{\left[\lambda_{k} / \tau\right]-1}-R_{5} R_{6} R^{\left[\lambda_{k} / \tau\right]-1}\right) \\
& \left.\left.\cdot\left|\frac{\lambda_{k}}{\tau}-\left[\frac{\lambda_{k}}{\tau}\right]\right|^{3}\right) f_{1}\right]+\frac{1}{2} R_{4} \tau^{2} \sum_{k=1}^{n} \alpha_{k} \\
& \cdot\left[\sum _ { s = 2 } ^ { [ \lambda _ { k } / \tau ] - 1 } \left(\left(R_{6} R^{\left[\lambda_{k} / \tau\right]-s}-\widetilde{R}_{6} \widetilde{R}^{\left[\lambda_{k} / \tau\right]-s}\right)\right.\right.
\end{aligned}
$$

$$
\begin{aligned}
& -\frac{3}{2} A \tau^{2}\left(R_{6} R^{\left[\lambda_{k} / \tau\right]-s}-\widetilde{R}_{6} \widetilde{R}^{\left[\lambda_{k} / \tau\right]-s}\right) \\
& \left.\cdot\left|\frac{\lambda_{k}}{\tau}-\left[\frac{\lambda_{k}}{\tau}\right]\right|^{2}\right)\left(f_{s}-f_{s-1}\right) \\
& +\left(\left(\widetilde{R}_{6}-R_{6}\right)-\frac{3}{2} A \tau^{2}\left(\widetilde{R}_{6}-R_{6}\right)\left|\frac{\lambda_{k}}{\tau}-\left[\frac{\lambda_{k}}{\tau}\right]\right|^{2}\right) f_{\left[\lambda_{k} / \tau\right]-1} \\
& -\left(\left(\widetilde{R}_{6} \widetilde{R}^{\left[\lambda_{k} / \tau\right]-1}-R_{6} R^{\left[\lambda_{k} / \tau\right]-1}\right)\right. \\
& \left.\left.+\frac{3}{2} A \tau^{2}\left(\widetilde{R}_{6} \widetilde{R}^{\left[\lambda_{k} / \tau\right]-1}-R_{6} R^{\left[\lambda_{k} / \tau\right]-1}\right)\left|\frac{\lambda_{k}}{\tau}-\left[\frac{\lambda_{k}}{\tau}\right]\right|^{2}\right) f_{1}\right] \\
& \cdot \frac{1}{2} R_{4} \sum_{k=1}^{n} \alpha_{k} R_{7}\left\{\left|\frac{\lambda_{k}}{\tau}-\left[\frac{\lambda_{k}}{\tau}\right]\right|-\frac{7}{6} A \tau^{2}\left|\frac{\lambda_{k}}{\tau}-\left[\frac{\lambda_{k}}{\tau}\right]\right|^{3}\right\} \\
& \cdot f_{\left[\lambda_{k} / \tau\right]-1} \tau^{2}+\sum_{k=1}^{n} \alpha_{k}\left\{\frac{3}{2}\left|\frac{\lambda_{k}}{\tau}-\left[\frac{\lambda_{k}}{\tau}\right]\right|^{2} f_{\left[\lambda_{k} / \tau\right]} \tau^{2}\right. \\
& \left.\left.+\frac{7}{6}\left|\frac{\lambda_{k}}{\tau}-\left[\frac{\lambda_{k}}{\tau}\right]\right|^{3} f_{\left[\lambda_{k} / \tau\right]}^{\prime} \tau^{3}\right\}+\varphi\right) \\
& -\left(-\frac{1}{2} R_{2} \sum_{k=1}^{n} \alpha_{k}\right. \\
& \cdot\left\{\left(\widetilde{R}_{1} R^{\left[\lambda_{k} / \tau\right]-1} R_{5}+R_{1} \widetilde{R}^{\left[\lambda_{k} / \tau\right]-1} \widetilde{R}_{5}\right)\left|\frac{\lambda_{k}}{\tau}-\left[\frac{\lambda_{k}}{\tau}\right]\right|\right. \\
& -\frac{7}{6} \tau^{2} A\left(\widetilde{R}_{1} R^{\left[\lambda_{k} / \tau\right]-1} R_{5}+R_{1} \widetilde{R}^{\left[\lambda_{k} / \tau\right]-1} \widetilde{R}_{5}\right)\left|\frac{\lambda_{k}}{\tau}-\left[\frac{\lambda_{k}}{\tau}\right]\right|^{3} \\
& +\left(\widetilde{R}_{1} R^{\left[\lambda_{k} / \tau\right]}+R_{1} \widetilde{R}^{\left[\lambda_{k} / \tau\right]}\right) \\
& \left.\left.-\frac{3}{2} A \tau^{2}\left(\widetilde{R}_{1} R^{\left[\lambda_{k} / \tau\right]}+R_{1} \widetilde{R}^{\left[\lambda_{k} / \tau\right]}\right)\left|\frac{\lambda_{k}}{\tau}-\left[\frac{\lambda_{k}}{\tau}\right]^{2}\right|\right\}\right) \\
& \cdot\left(R_{3} \sum_{k=1}^{n} \frac{1}{2} \beta_{k} f_{1,1} \tau^{2}\right. \\
& \left\{\tau^{-1}\left(\widetilde{R}^{\left[\lambda_{k} / \tau\right]-1} \widetilde{R}_{5}+R^{\left[\lambda_{k} / \tau\right]-1} R_{5}\right)\right. \\
& -\frac{1}{2} \tau A\left(\widetilde{R}^{\left[\lambda_{k} / \tau\right]-1} \widetilde{R}_{5}+R^{\left[\lambda_{k} / \tau\right]-1} R_{5}\right)\left|\frac{\lambda_{k}}{\tau}-\left[\frac{\lambda_{k}}{\tau}\right]\right|^{2} \\
& -A \tau\left(\widetilde{R}^{\left[\lambda_{k} / \tau\right]}+R^{\left[\lambda_{k} / \tau\right]}\right)\left|\frac{\lambda_{k}}{\tau}-\left[\frac{\lambda_{k}}{\tau}\right]\right| \\
& \left.+\frac{1}{6} A^{2} \tau^{3}\left(\widetilde{R}^{\left[\lambda_{k} / \tau\right]}+R^{\left[\lambda_{k} / \tau\right]}\right)\left|\frac{\lambda_{k}}{\tau}-\left[\frac{\lambda_{k}}{\tau}\right]\right|^{3}\right\} \\
& +\frac{1}{2} R_{4} \tau^{2} \sum_{k=1}^{n} \beta_{k} \\
& \cdot\left[\sum _ { s = 2 } ^ { [ \lambda _ { k } / \tau ] - 2 } \left(\frac{1}{\tau}\left(R_{5} R_{6} R^{\left[\lambda_{k} / \tau\right]-s-1}-\widetilde{R}_{5} \widetilde{R}_{6} \widetilde{R}^{\left[\lambda_{k} / \tau\right]-s-1}\right)\right.\right. \\
& -\frac{1}{2} A \tau\left(R_{5} R_{6} R^{\left[\lambda_{k} / \tau\right]-s-1}-\widetilde{R}_{5} \widetilde{R}_{6} \widetilde{R}^{\left[\lambda_{k} / \tau\right]-s-1}\right)
\end{aligned}
$$




$$
\begin{aligned}
& \left.\cdot\left|\frac{\lambda_{k}}{\tau}-\left[\frac{\lambda_{k}}{\tau}\right]\right|^{2}\right)\left(f_{s}-f_{s-1}\right) \\
& \left\{\left(\widetilde{R}_{1} R^{\left[\lambda_{k} / \tau\right]-1} R_{5}+R_{1} \widetilde{R}^{\left[\lambda_{k} / \tau\right]-1} \widetilde{R}_{5}\right)\right. \\
& +\left(\frac{1}{\tau}\left(\widetilde{R}_{5} \widetilde{R}_{6}-R_{5} R_{6}\right)-\frac{1}{2} A \tau\left|\frac{\lambda_{k}}{\tau}-\left[\frac{\lambda_{k}}{\tau}\right]\right|^{2}\right. \\
& \cdot\left|\frac{\lambda_{k}}{\tau}-\left[\frac{\lambda_{k}}{\tau}\right]\right| \\
& -\frac{7}{6} A \tau^{2}\left(\widetilde{R}_{1} R^{\left[\lambda_{k} / \tau\right]-1} R_{5}+R_{1} \widetilde{R}^{\left[\lambda_{k} / \tau\right]-1} \widetilde{R}_{5}\right) \\
& \cdot\left|\frac{\lambda_{k}}{\tau}-\left[\frac{\lambda_{k}}{\tau}\right]\right|^{3} \\
& +\left(\widetilde{R}_{1} R^{\left[\lambda_{k} / \tau\right]}+R_{1} \widetilde{R}^{\left[\lambda_{k} / \tau\right]}\right) \\
& \left.\left.\cdot\left(1-\frac{3}{2} A \tau^{2}\left|\frac{\lambda_{k}}{\tau}-\left[\frac{\lambda_{k}}{\tau}\right]\right|^{2}\right)\right\}\right) \\
& \left.\left.\cdot\left|\frac{\lambda_{k}}{\tau}-\left[\frac{\lambda_{k}}{\tau}\right]\right|^{2}\right) f_{1}\right] \\
& +\frac{1}{2} R_{4} \tau^{2} \sum_{k=1}^{n} \beta_{k} \\
& \cdot\left[\sum _ { s = 2 } ^ { [ \lambda _ { k } / \tau ] - 1 } \left(-A \tau\left(R_{6} R^{\left[\lambda_{k} / \tau\right]-s}-\widetilde{R}_{6} \widetilde{R}^{\left[\lambda_{k} / \tau\right]-s}\right)\right.\right. \\
& \cdot\left|\frac{\lambda_{k}}{\tau}-\left[\frac{\lambda_{k}}{\tau}\right]\right|+\frac{1}{6} A \tau^{2}\left(R_{6} R^{\left[\lambda_{k} / \tau\right]-s}-\widetilde{R}_{6} \widetilde{R}^{\left[\lambda_{k} / \tau\right]-s}\right) \\
& \left.\cdot\left|\frac{\lambda_{k}}{\tau}-\left[\frac{\lambda_{k}}{\tau}\right]\right|^{3}\right)\left(f_{s}-f_{s-1}\right) \\
& +\left(-A \tau\left(\widetilde{R}_{6}-R_{6}\right)\left|\frac{\lambda_{k}}{\tau}-\left[\frac{\lambda_{k}}{\tau}\right]\right|\right. \\
& \left.+\frac{1}{6} A \tau^{2}\left(\widetilde{R}_{6}-R_{6}\right)\left|\frac{\lambda_{k}}{\tau}-\left[\frac{\lambda_{k}}{\tau}\right]\right|^{3}\right) f_{\left[\lambda_{k} / \tau\right]-1} \\
& -\left(-A \tau\left(\widetilde{R}_{6} \widetilde{R}^{\left[\lambda_{k} / \tau\right]-1}-R_{6} R^{\left[\lambda_{k} / \tau\right]-1}\right)\left|\frac{\lambda_{k}}{\tau}-\left[\frac{\lambda_{k}}{\tau}\right]\right|\right. \\
& \left.\left.+\frac{1}{6} A \tau^{2}\left(\widetilde{R}_{6} \widetilde{R}^{\left[\lambda_{k} / \tau\right]-1}-R_{6} R^{\left[\lambda_{k} / \tau\right]-1}\right)\left|\frac{\lambda_{k}}{\tau}-\left[\frac{\lambda_{k}}{\tau}\right]\right|^{3}\right) f_{1}\right] \\
& +\frac{1}{2} \sum_{k=1}^{n} R_{4} \beta_{k} R_{7}\left(\frac{1}{\tau}-\frac{1}{2} A \tau\left|\frac{\lambda_{k}}{\tau}-\left[\frac{\lambda_{k}}{\tau}\right]\right|^{2}\right) f_{\left[\lambda_{k} / \tau\right]-1} \tau^{2} \\
& +\sum_{k=1}^{n} \beta_{k} \\
& \cdot\left\{-\frac{1}{6} A \tau^{3}\left|\frac{\lambda_{k}}{\tau}-\left[\frac{\lambda_{k}}{\tau}\right]\right|^{3} f_{\left[\lambda_{k} / \tau\right]}+\left|\frac{\lambda_{k}}{\tau}-\left[\frac{\lambda_{k}}{\tau}\right]\right| f_{\left[\lambda_{k} / \tau\right]} \tau\right. \\
& +\frac{1}{2}\left|\frac{\lambda_{k}}{\tau}-\left[\frac{\lambda_{k}}{\tau}\right]\right|^{2} f_{\left[\lambda_{k} / \tau\right]}^{\prime} \tau^{2} \\
& \left.\left.\left.+\frac{1}{6} \tau^{3}\left|\frac{\lambda_{k}}{\tau}-\left[\frac{\lambda_{k}}{\tau}\right]\right|^{3} f_{\left[\lambda_{k} / \tau\right]}^{\prime \prime}\right\}+\psi\right)\right\}, \\
& \cdot\left(R_{3} \sum_{k=1}^{n} \frac{1}{2} \beta_{k} f_{1,1} \tau^{2}\right. \\
& \cdot\left\{\tau^{-1}\left(\widetilde{R}^{\left[\lambda_{k} / \tau\right]-1} \widetilde{R}_{5}+R^{\left[\lambda_{k} / \tau\right]-1} R_{5}\right)\right. \\
& -\frac{1}{2} \tau A\left(\widetilde{R}^{\left[\lambda_{k} / \tau\right]-1} \widetilde{R}_{5}+R^{\left[\lambda_{k} / \tau\right]-1} R_{5}\right) \\
& \cdot\left|\frac{\lambda_{k}}{\tau}-\left[\frac{\lambda_{k}}{\tau}\right]\right|^{2} \\
& -A \tau\left(\widetilde{R}^{\left[\lambda_{k} / \tau\right]}+R^{\left[\lambda_{k} / \tau\right]}\right)\left|\frac{\lambda_{k}}{\tau}-\left[\frac{\lambda_{k}}{\tau}\right]\right| \\
& \left.+\frac{1}{6} A^{2} \tau^{3}\left(\widetilde{R}^{\left[\lambda_{k} / \tau\right]}+R^{\left[\lambda_{k} / \tau\right]}\right)\left|\frac{\lambda_{k}}{\tau}-\left[\frac{\lambda_{k}}{\tau}\right]\right|^{3}\right\} \\
& +\frac{1}{2} R_{4} \tau^{2} \sum_{k=1}^{n} \beta_{k} \\
& \cdot\left[\sum _ { s = 2 } ^ { [ \lambda _ { k } / \tau ] - 2 } \left(\frac { 1 } { \tau } \left(R_{5} R_{6} R^{\left[\lambda_{k} / \tau\right]-s-1}\right.\right.\right. \\
& \left.-\widetilde{R}_{5} \widetilde{R}_{6} \widetilde{R}^{\left[\lambda_{k} / \tau\right]-s-1}\right) \\
& -\frac{1}{2} A \tau\left(R_{5} R_{6} R^{\left[\lambda_{k} / \tau\right]-s-1}-\widetilde{R}_{5} \widetilde{R}_{6} \widetilde{R}^{\left[\lambda_{k} / \tau\right]-s-1}\right) \\
& \left.\cdot\left|\frac{\lambda_{k}}{\tau}-\left[\frac{\lambda_{k}}{\tau}\right]\right|^{2}\right)\left(f_{s}-f_{s-1}\right) \\
& +\left(\frac{1}{\tau}\left(\widetilde{R}_{5} \widetilde{R}_{6}-R_{5} R_{6}\right)\right. \\
& -\frac{1}{2} A \tau\left|\frac{\lambda_{k}}{\tau}-\left[\frac{\lambda_{k}}{\tau}\right]\right|^{2} \\
& \left.\cdot\left(\widetilde{R}_{5} \widetilde{R}_{6}-R_{5} R_{6}\right)\left|\frac{\lambda_{k}}{\tau}-\left[\frac{\lambda_{k}}{\tau}\right]\right|^{2}\right) f_{\left[\lambda_{k} / \tau\right]-2} \\
& -\left(\frac{1}{\tau}\left(\widetilde{R}_{5} \widetilde{R}_{6} \widetilde{R}^{\left[\lambda_{k} / \tau\right]-1}-R_{5} R_{6} R^{\left[\lambda_{k} / \tau\right]-1}\right)\right. \\
& -\frac{1}{2} A \tau\left(\widetilde{R}_{5} \widetilde{R}_{6} \widetilde{R}^{\left[\lambda_{k} / \tau\right]-1}-R_{5} R_{6} R^{\left[\lambda_{k} / \tau\right]-1}\right) \\
& \cdot\left\{\left(1-\frac{1}{2} \sum_{k=1}^{n} a_{k}\right.\right. \\
& \omega=T_{\tau}\left(I+i \tau^{2} A^{4}\right)^{-1} \\
& \left.\left.\cdot\left|\frac{\lambda_{k}}{\tau}-\left[\frac{\lambda_{k}}{\tau}\right]\right|^{2}\right) f_{1}\right]
\end{aligned}
$$




$$
\begin{aligned}
& +\frac{1}{2} R_{4} \tau^{2} \sum_{k=1}^{n} \beta_{k} \\
& \cdot\left(\sum_{k=1}^{n} \frac{1}{2} \alpha_{k} R_{3} \tau^{2} f_{1,1}\right. \\
& \cdot\left[\sum _ { s = 2 } ^ { [ \lambda _ { k } / \tau ] - 1 } \left(-A \tau\left(R_{6} R^{\left[\lambda_{k} / \tau\right]-s}-\widetilde{R}_{6} \widetilde{R}^{\left[\lambda_{k} / \tau\right]-s}\right)\right.\right. \\
& \left\{\left(\widetilde{R}^{\left[\lambda_{k} / \tau\right]-1} \widetilde{R}_{5}+R^{\left[\lambda_{k} / \tau\right]-1} R_{5}\right)\left|\frac{\lambda_{k}}{\tau}-\left[\frac{\lambda_{k}}{\tau}\right]\right|\right. \\
& \cdot\left|\frac{\lambda_{k}}{\tau}-\left[\frac{\lambda_{k}}{\tau}\right]\right| \\
& +\frac{7}{6} A \tau^{2}\left(R^{\left[\lambda_{k} / \tau\right]-1} R_{5}+\widetilde{R}^{\left[\lambda_{k} / \tau\right]-1} \widetilde{R}_{5}\right)\left|\frac{\lambda_{k}}{\tau}-\left[\frac{\lambda_{k}}{\tau}\right]\right|^{3} \\
& \left.+\frac{1}{6} A \tau^{2}\left(R_{6} R^{\left[\lambda_{k} / \tau\right]-s}-\widetilde{R}_{6} \widetilde{R}^{\left[\lambda_{k} / \tau\right]-s}\right)\left|\frac{\lambda_{k}}{\tau}-\left[\frac{\lambda_{k}}{\tau}\right]\right|^{3}\right) \\
& +\left[\widetilde{R}^{\left[\lambda_{k} / \tau\right]}+R^{\left[\lambda_{k} / \tau\right]}\right] \\
& \cdot\left(f_{s}-f_{s-1}\right) \\
& -\frac{3}{2} \tau^{2} A\left[\widetilde{R}^{\left[\lambda_{k} / \tau\right]}+R^{\left[\lambda_{k} / \tau\right]}\right] \\
& +\left(-A \tau\left(\widetilde{R}_{6}-R_{6}\right)\left|\frac{\lambda_{k}}{\tau}-\left[\frac{\lambda_{k}}{\tau}\right]\right|\right. \\
& \left.+\frac{1}{6} A \tau^{2}\left(\widetilde{R}_{6}-R_{6}\right)\left|\frac{\lambda_{k}}{\tau}-\left[\frac{\lambda_{k}}{\tau}\right]\right|^{3}\right) f_{\left[\lambda_{k} / \tau\right]-1} \\
& -\left(-A \tau\left(\widetilde{R}_{6} \widetilde{R}^{\left[\lambda_{k} / \tau\right]-1}-R_{6} R^{\left[\lambda_{k} / \tau\right]-1}\right)\left|\frac{\lambda_{k}}{\tau}-\left[\frac{\lambda_{k}}{\tau}\right]\right|\right. \\
& \left.\cdot\left|\frac{\lambda_{k}}{\tau}-\left[\frac{\lambda_{k}}{\tau}\right]\right|^{2}\right\} \\
& +\frac{1}{2} R_{4} \tau^{2} \sum_{k=1}^{n} \alpha_{k} \\
& \cdot\left[\sum _ { s = 2 } ^ { [ \lambda _ { k } / \tau ] - 2 } \left(\left(R_{5} R_{6} R^{\left[\lambda_{k} / \tau\right]-s-1}-\widetilde{R}_{5} \widetilde{R}_{6} \widetilde{R}^{\left[\lambda_{k} / \tau\right]-s-1}\right)\right.\right. \\
& +\frac{1}{6} A \tau^{2}\left(\widetilde{R}_{6} \widetilde{R}^{\left[\lambda_{k} / \tau\right]-1}-R_{6} R^{\left[\lambda_{k} / \tau\right]-1}\right) \\
& \cdot\left|\frac{\lambda_{k}}{\tau}-\left[\frac{\lambda_{k}}{\tau}\right]\right| \\
& \left.\left.\left|\frac{\lambda_{k}}{\tau}-\left[\frac{\lambda_{k}}{\tau}\right]\right|^{3}\right) f_{1}\right] \\
& +\frac{1}{2} \sum_{k=1}^{n} R_{4} \beta_{k} R_{7}\left(\frac{1}{\tau}-\frac{1}{2} A \tau\left|\frac{\lambda_{k}}{\tau}-\left[\frac{\lambda_{k}}{\tau}\right]\right|^{2}\right) f_{\left[\lambda_{k} / \tau\right]-1} \tau^{2} \\
& +\sum_{k=1}^{n} \beta_{k}\left\{-\frac{1}{6} A \tau^{3}\left|\frac{\lambda_{k}}{\tau}-\left[\frac{\lambda_{k}}{\tau}\right]\right|^{3} f_{\left[\lambda_{k} / \tau\right]}\right. \\
& +\left|\frac{\lambda_{k}}{\tau}-\left[\frac{\lambda_{k}}{\tau}\right]\right| f_{\left[\lambda_{k} / \tau\right]} \tau \\
& +\frac{1}{2}\left|\frac{\lambda_{k}}{\tau}-\left[\frac{\lambda_{k}}{\tau}\right]\right|^{2} f_{\left[\lambda_{k} / \tau\right]}^{\prime} \tau^{2} \\
& \left.\left.+\frac{1}{6} \tau^{3}\left|\frac{\lambda_{k}}{\tau}-\left[\frac{\lambda_{k}}{\tau}\right]\right|^{3} f_{\left[\lambda_{k} / \tau\right]}^{\prime \prime}\right\}+\psi\right) \\
& -\left(-\frac{1}{2} \sum_{k=1}^{n} \beta_{k}\right. \\
& \cdot\left\{\frac{1}{\tau}\left(\widetilde{R}_{1} R^{\left[\lambda_{k} / \tau\right]-1} R_{5}+R_{1} \widetilde{R}^{\left[\lambda_{k} / \tau\right]-1} \widetilde{R}_{5}\right)\right. \\
& +\frac{1}{2} A \tau\left(\widetilde{R}_{1} R^{\left[\lambda_{k} / \tau\right]-1} R_{5}+R_{1} \widetilde{R}^{\left[\lambda_{k} / \tau\right]-1} \widetilde{R}_{5}\right) \\
& \cdot\left|\frac{\lambda_{k}}{\tau}-\left[\frac{\lambda_{k}}{\tau}\right]\right|^{2} \\
& -A \tau\left(\widetilde{R}_{1} R^{\left[\lambda_{k} / \tau\right]}+R_{1} \widetilde{R}^{\left[\lambda_{k} / \tau\right]}\right) \\
& \cdot\left|\frac{\lambda_{k}}{\tau}-\left[\frac{\lambda_{k}}{\tau}\right]\right| \\
& \left.\left.+\frac{1}{6} A^{2} \tau^{3}\left(\widetilde{R}_{1} R^{\left[\lambda_{k} / \tau\right]}+R_{1} \widetilde{R}^{\left[\lambda_{k} / \tau\right]}\right)\left|\frac{\lambda_{k}}{\tau}-\left[\frac{\lambda_{k}}{\tau}\right]\right|^{3}\right\}\right) \\
& -\frac{7}{6} A \tau^{2}\left(R_{5} R_{6} R^{\left[\lambda_{k} / \tau\right]-s-1}\right. \\
& \left.\left.-\widetilde{R}_{5} \widetilde{R}_{6} \widetilde{R}^{\left[\lambda_{k} / \tau\right]-s-1}\right)\left|\frac{\lambda_{k}}{\tau}-\left[\frac{\lambda_{k}}{\tau}\right]\right|^{3}\right) \\
& \cdot\left(f_{s}-f_{s-1}\right) \\
& +\left(\left(\widetilde{R}_{5} \widetilde{R}_{6}-R_{5} R_{6}\right)\right. \\
& \cdot\left|\frac{\lambda_{k}}{\tau}-\left[\frac{\lambda_{k}}{\tau}\right]\right| \\
& -\frac{7}{6} A \tau^{2}\left(\widetilde{R}_{5} \widetilde{R}_{6}-R_{5} R_{6}\right) \\
& \left.\cdot\left|\frac{\lambda_{k}}{\tau}-\left[\frac{\lambda_{k}}{\tau}\right]\right|^{3}\right) f_{\left[\lambda_{k} / \tau\right]-2} \\
& -\left(\left(\widetilde{R}_{5} \widetilde{R}_{6} \widetilde{R}^{\left[\lambda_{k} / \tau\right]-1}-R_{5} R_{6} R^{\left[\lambda_{k} / \tau\right]-1}\right)\right. \\
& \cdot\left|\frac{\lambda_{k}}{\tau}-\left[\frac{\lambda_{k}}{\tau}\right]\right|-\frac{7}{6} A \tau^{2} \\
& \cdot\left(\widetilde{R}_{5} \widetilde{R}_{6} \widetilde{R}^{\left[\lambda_{k} / \tau\right]-1}-R_{5} R_{6} R^{\left[\lambda_{k} / \tau\right]-1}\right) \\
& \left.\left.\cdot\left|\frac{\lambda_{k}}{\tau}-\left[\frac{\lambda_{k}}{\tau}\right]\right|^{3}\right) f_{1}\right]+\frac{1}{2} R_{4} \tau^{2} \sum_{k=1}^{n} \alpha_{k} \\
& \cdot\left[\sum _ { s = 2 } ^ { [ \lambda _ { k } / \tau ] - 1 } \left(\left(R_{6} R^{\left[\lambda_{k} / \tau\right]-s}-\widetilde{R}_{6} \widetilde{R}^{\left[\lambda_{k} / \tau\right]-s}\right)\right.\right. \\
& -\frac{3}{2} A \tau^{2}\left(R_{6} R^{\left[\lambda_{k} / \tau\right]-s}-\widetilde{R}_{6} \widetilde{R}^{\left[\lambda_{k} / \tau\right]-s}\right) \\
& \left.\cdot\left|\frac{\lambda_{k}}{\tau}-\left[\frac{\lambda_{k}}{\tau}\right]\right|^{2}\right)\left(f_{s}-f_{s-1}\right)
\end{aligned}
$$




$$
\begin{aligned}
& +\left(\left(\widetilde{R}_{6}-R_{6}\right)-\frac{3}{2} A \tau^{2}\left(\widetilde{R}_{6}-R_{6}\right)\right. \\
& \left.\cdot\left|\frac{\lambda_{k}}{\tau}-\left[\frac{\lambda_{k}}{\tau}\right]\right|^{2}\right) f_{\left[\lambda_{k} / \tau\right]-1} \\
& -\left(\left(\widetilde{R}_{6} \widetilde{R}^{\left[\lambda_{k} / \tau\right]-1}-R_{6} R^{\left[\lambda_{k} / \tau\right]-1}\right)-\frac{3}{2} A \tau^{2}\right. \\
& \cdot\left(\widetilde{R}_{6} \widetilde{R}^{\left[\lambda_{k} / \tau\right]-1}-R_{6} R^{\left[\lambda_{k} / \tau\right]-1}\right) \\
& \left.\left.\cdot\left|\frac{\lambda_{k}}{\tau}-\left[\frac{\lambda_{k}}{\tau}\right]\right|^{2}\right) f_{1}\right] \\
& \cdot \frac{1}{2} R_{4} \sum_{k=1}^{n} \alpha_{k} R_{7} \\
& \cdot\left\{\left|\frac{\lambda_{k}}{\tau}-\left[\frac{\lambda_{k}}{\tau}\right]\right|\right. \\
& \left.-\frac{7}{6} A \tau^{2}\left|\frac{\lambda_{k}}{\tau}-\left[\frac{\lambda_{k}}{\tau}\right]\right|^{3}\right\} f_{\left[\lambda_{k} / \tau\right]-1} \tau^{2} \\
& +\sum_{k=1}^{n} \alpha_{k}\left\{\frac{3}{2}\left|\frac{\lambda_{k}}{\tau}-\left[\frac{\lambda_{k}}{\tau}\right]\right|^{2} f_{\left[\lambda_{k} / \tau\right]} \tau^{2}\right. \\
& \left.\left.+\frac{7}{6}\left|\frac{\lambda_{k}}{\tau}-\left[\frac{\lambda_{k}}{\tau}\right]\right|^{3} f_{\left[\lambda_{k} / \tau \tau\right.}^{\prime} \tau^{3}+\varphi\right)\right\}
\end{aligned}
$$

Let us obtain estimates for $\|A \mu\|_{H}$ and $\left\|A^{1 / 2} \omega\right\|_{H}$ using the method of lemmas. First, applying $A$ to formula (24), using estimates (11) and (15) and the triangle inequality, we obtain

$$
\begin{gathered}
\|A \mu\|_{H} \leq M\left\{\sum_{s=2}^{N-1}\left\|f_{s}-f_{s-1}\right\|_{H}+\left\|f_{1}\right\|_{H}+\|A \varphi\|_{H}\right. \\
\left.+\left\|A^{1 / 2} \psi\right\|_{H}+\tau\left\|A^{1 / 2} f_{1,1}\right\|_{H}\right\} .
\end{gathered}
$$

Second, applying $A^{1 / 2}$ to formula (25), using estimates (11) and (15) and the triangle inequality, we obtain

$$
\begin{aligned}
& \left\|A^{1 / 2} \omega\right\|_{H} \leq M\left\{\sum_{s=2}^{N-1}\left\|f_{s}-f_{s-1}\right\|_{H}+\left\|f_{1}\right\|_{H}+\|A \varphi\|_{H}\right. \\
& \left.+\left\|A^{1 / 2} \psi\right\|_{H}+\tau\left\|A^{1 / 2} f_{1,1}\right\|_{H}\right\} .
\end{aligned}
$$

The estimates (21) are proved for $k=0,1$. Now, we give the proofs of estimates (21) which were also presented in [14] for $k \geq 2$. Using formula (19), estimates (11), (22), and (23), the triangle inequality, and method of Lemmas 1 and 2, we obtain

$$
\begin{gathered}
\left\|u_{k}\right\|_{H} \leq M\left\{\sum_{s=1}^{N-1}\left\|A^{-1 / 2} f_{s}\right\|_{H} \tau+\|\varphi\|_{H}+\left\|A^{-1 / 2} \psi\right\|_{H}\right. \\
\left.+\tau\left\|A^{-1 / 2} f_{1,1}\right\|_{H}\right\} .
\end{gathered}
$$

Applying $A^{1 / 2}$ to (19), we get

$$
\begin{aligned}
& \left\|A^{1 / 2} u_{k}\right\|_{H} \leq M\left\{\sum_{s=1}^{N-1}\left\|f_{s}\right\|_{H} \tau\right. \\
& \left.\quad+\left\|A^{1 / 2}\left(I+i \tau A^{1 / 2}\right) \varphi\right\|_{H}+\|\psi\|_{H}+\tau\left\|f_{1,1}\right\|_{H}\right\} .
\end{aligned}
$$

Applying Abel's formula to (19), we get

$$
\begin{aligned}
u_{k} & =\frac{1}{2}\left[\widetilde{R}_{1} R^{k}-R_{1} \widetilde{R}^{k}\right] \mu+\frac{1}{2}\left[\widetilde{R}^{k}-R^{k}\right] R_{2} \omega+\frac{1}{2}\left[\widetilde{R}^{k}\right. \\
& \left.-R^{k}\right] R_{3} \tau^{2} f_{1,1}+\tau^{2} R_{4} \\
& \cdot \frac{1}{2}\left(\sum_{s=2}^{k-1}\left[R_{6} R^{k-s}-\widetilde{R}_{6} \widetilde{R}^{k-s}\right]\left(f_{s}-f_{s-1}\right)\right. \\
& \left.+\left(\widetilde{R}_{6}-R_{6}\right) f_{k-1}-\left[\widetilde{R}_{6} \widetilde{R}^{k-1}-R_{6} R^{k-1}\right] f_{1}\right), \\
& 2 \leq k \leq N .
\end{aligned}
$$

Applying $A$ to formula (30), using estimates (11) and the triangle inequality, we obtain

$$
\begin{gathered}
\left\|A u_{k}\right\|_{H} \leq M\left\{\sum_{s=2}^{N-1}\left\|f_{s}-f_{s-1}\right\|_{H}+\left\|f_{1}\right\|_{H}\right. \\
+\left\|A\left(I+i \tau A^{1 / 2}\right) \varphi\right\|_{H}+\left\|A^{1 / 2} \psi\right\|_{H} \\
\left.+\tau\left\|A^{1 / 2} f_{1,1}\right\|_{H}\right\} .
\end{gathered}
$$

Theorem 3 is proved.

We denote the mesh function of approximation by $a^{\tau}=$ $\left\{a_{k}\right\}_{k=0}^{N}$. Then $\left\|\left(I+\tau^{2} A^{4}\right)^{-1} a_{1}\right\|_{H} \sim\left\|a_{1}\right\|_{H}=o(\tau)$ if we assume that $\tau\left\|A a_{1}\right\|_{H}$ tends to 0 as $\tau \rightarrow 0$ not slower than $\left\|a_{1}\right\|_{H}$. It takes place in applications by supplementary restriction of the smooth property of the data of space variables. Let us give applications of the abstract results presented in Theorem 3. tion

First, the mixed multipoint NBVP for hyperbolic equa-

$$
\begin{aligned}
u_{t t}-\left(a(x) u_{x}\right)_{x}+\delta u & =f(t, x), \\
0<t<1,0<x<1, & \\
u(0, x)=\sum_{j=1}^{n} \alpha_{j} u\left(\lambda_{j}, x\right)+\varphi(x), & 0 \leq x \leq 1,
\end{aligned}
$$




$$
\begin{gathered}
u(t, 0)=u(t, 1), \\
u_{x}(t, 0)=u_{x}(t, 1),
\end{gathered}
$$$$
u_{t}(0, x)=\sum_{j=1}^{n} \beta_{j} u_{t}\left(\lambda_{j}, x\right)+\psi(x),
$$$$
0 \leq x \leq 1
$$$$
0 \leq t \leq 1
$$

is considered. Problem (32) has a unique smooth solution $u(t, x), \delta>0$, and the smooth functions $a(x) \geq a>0$ $(a(0)=a(1), x \in(0,1)), \varphi(x), \psi(x)(x \in[0,1])$ and $f(t, x)$ $(t, x \in[0,1])$. This allows us to reduce mixed problem (32) to multipoint NBVP (1) in a Hilbert space $H=L_{2}[0,1]$ with a self-adjoint positive definite operator $A^{x}$ defined by (32).

The discretization of problem (32) is carried out in two steps. In the first step, we present the grid space

$$
[0,1]_{h}=\left\{x: x_{r}=r h, 0 \leq r \leq K, K h=1\right\}
$$

in a Hilbert space $L_{2 h}=L_{2}\left([0,1]_{h}\right), W_{2 h}^{1}=W_{2 h}^{1}\left([0,1]_{h}\right)$ and $W_{2 h}^{2}=W_{2 h}^{2}\left([0,1]_{h}\right)$ of the grid functions $\varphi^{h}(x)=\left\{\varphi_{r}\right\}_{1}^{K-1}$ defined on $[0,1]_{h}$, equipped with the norms

$$
\begin{aligned}
\left\|\varphi^{h}\right\|_{L_{2 h}}= & \left(\sum_{r=1}^{K-1}\left|\varphi^{h}(x)\right|^{2} h\right)^{1 / 2}, \\
\left\|\varphi^{h}\right\|_{W_{2 h}^{1}}= & \left\|\varphi^{h}\right\|_{L_{2 h}}+\left(\sum_{r=1}^{K-1}\left|\left(\varphi^{h}\right)_{\bar{x}, j}\right|^{2} h\right)^{1 / 2}, \\
\left\|\varphi^{h}\right\|_{W_{2 h}^{2}}= & \left\|\varphi^{h}\right\|_{L_{2 h}}+\left(\sum_{r=1}^{K-1}\left|\left(\varphi^{h}\right)_{\bar{x}, j}\right|^{2} h\right)^{1 / 2} \\
& +\left(\sum_{r=1}^{K-1}\left|\left(\varphi^{h}\right)_{x \bar{x}, j}\right|^{2} h\right)^{1 / 2},
\end{aligned}
$$

respectively. To the differential operator $A$ generated by the problem (32), we assign the difference operator $A_{h}^{x}$ by the formula

$$
A_{h}^{x} \varphi^{h}(x)=\left\{-\left(a(x) \varphi_{\bar{x}}\right)_{x, r}+\delta \varphi_{r}\right\}_{1}^{K-1}
$$

acting in the space of grid functions $\varphi^{h}(x)=\left\{\varphi_{r}\right\}_{0}^{K}$ satisfying the conditions $\varphi_{0}=\varphi_{K}, \varphi_{1}-\varphi_{0}=\varphi_{K}-\varphi_{K-1}$. With the help of $A_{h}^{x}$ we arrive at the multipoint NBVP

$$
\frac{d^{2} v^{h}(t, x)}{d t^{2}}+A_{h}^{x} v^{h}(t, x)=f^{h}(t, x),
$$$$
0<t<1, x \in[0,1]_{h},
$$

$$
\begin{array}{r}
v^{h}(0, x)=\sum_{j=1}^{n} \alpha_{j} v^{h}\left(\lambda_{j}, x\right)+\varphi^{h}(x), \\
x \in[0,1]_{h}, \\
v_{t}^{h}(0, x)=\sum_{j=1}^{n} \beta_{j} v_{t}^{h}\left(\lambda_{j}, x\right)+\psi^{h}(x),
\end{array}
$$$$
x \in[0,1]_{h}
$$

for an infinite system of ordinary differential equations.

In the second step, we replace problem (36) by difference scheme (37):

$$
\begin{aligned}
& \tau^{-2}\left(u_{k+1}^{h}(x)-2 u_{k}^{h}(x)+u_{k-1}^{h}(x)\right)+\frac{2}{3} A_{h}^{x} u_{k}^{h}(x)+\frac{1}{6} \\
& \cdot A_{h}^{x}\left(u_{k+1}^{h}(x)+u_{k-1}^{h}(x)\right)+\frac{1}{12} \tau^{2}\left(A_{h}^{x}\right)^{2} u_{k+1}^{h}(x) \\
& =f_{k}^{h}(x) \text {, } \\
& f_{k}^{h}(x)=\frac{2}{3} f^{h}\left(t_{k}, x\right)+\frac{1}{6}\left(f^{h}\left(t_{k+1}, x\right)+f^{h}\left(t_{k-1}, x\right)\right) \\
& -\frac{1}{12} \tau^{2}\left(-A f^{h}\left(t_{k+1}, x\right)+f_{t t}^{h}\left(t_{k+1}, x\right)\right) \text {, } \\
& x \in[0,1]_{h}, t_{k}=k \tau, N \tau=1,1 \leq k \leq N-1, \\
& u_{0}^{h}(x)=\sum_{j=1}^{n} \alpha_{j}\left\{u_{\left[\lambda_{j} / \tau\right]}^{h}(x)\right. \\
& +\tau^{-1}\left(u_{\left[\lambda_{j} / \tau\right]}^{h}(x)-u_{\left[\lambda_{j} / \tau\right]-1}^{h}(x)\right)\left(\lambda_{j}-\left[\frac{\lambda_{j}}{\tau}\right] \tau\right) \\
& +\frac{3}{2}\left(f_{\left[\lambda_{j} / \tau\right]}-A_{h}^{x} u_{\left[\lambda_{j} / \tau\right]}^{h}(x)\right)\left(\lambda_{j}-\left[\frac{\lambda_{j}}{\tau}\right] \tau\right)^{2} \\
& +\frac{7}{6}\left(f_{\left[\lambda_{j} / \tau\right]}^{\prime}-\tau^{-1} A_{h}^{x}\left(u_{\left[\lambda_{j} / \tau\right]}^{h}(x)-u_{\left[\lambda_{j} / \tau\right]-1}^{h}(x)\right)\right) \\
& \left.\cdot\left(\lambda_{j}-\left[\frac{\lambda_{j}}{\tau}\right] \tau\right)^{3}\right\}+\varphi^{h}(x), \quad x \in[0,1]_{h}, \\
& \left(I+\tau^{2}\left(A_{h}^{x}\right)^{4}\right) \tau^{-1}\left(u_{1}^{h}(x)-u_{0}^{h}(x)\right)=\sum_{j=1}^{n} \beta_{j} \\
& \left\{\tau^{-1}\left(u_{\left[\lambda_{j} / \tau\right]}^{h}(x)-u_{\left[\lambda_{j} / \tau\right]-1}^{h}(x)\right)\right. \\
& +\left(f_{\left[\lambda_{j} / \tau\right]}-A_{h}^{x} u_{\left[\lambda_{j} / \tau\right]}^{h}(x)\right)\left(\lambda_{j}-\left[\frac{\lambda_{j}}{\tau}\right] \tau\right) \\
& +\frac{1}{2 !}\left(f_{\left[\lambda_{j} / \tau\right]}^{\prime}-\tau^{-1} A_{h}^{x}\left(u_{\left[\lambda_{j} / \tau\right]}^{h}(x)-u_{\left[\lambda_{j} / \tau\right]-1}^{h}(x)\right)\right) \\
& \cdot\left(\lambda_{j}-\left[\frac{\lambda_{j}}{\tau}\right] \tau\right)^{2}
\end{aligned}
$$




$$
\begin{aligned}
& +\frac{1}{3 !}\left(f_{\left[\lambda_{j} / \tau\right]}^{\prime \prime}-A_{h}^{x} f_{\left[\lambda_{j} / \tau\right]}+\left(A_{h}^{x}\right)^{2} u_{\left[\lambda_{j} / \tau\right]}^{h}(x)\right) \\
& \left.\cdot\left(\lambda_{j}-\left[\frac{\lambda_{j}}{\tau}\right] \tau\right)^{3}\right\}+\psi^{h}(x), \quad x \in[0,1]_{h}, \\
& f_{1,1}^{h}(x)=\frac{1}{2} f^{h}(0, x)+\frac{\tau}{6} f_{t}^{h}(0, x) .
\end{aligned}
$$

Theorem 4. Let $\tau$ and $h$ be sufficiently small numbers. Then, the solution of difference scheme (37) satisfies the following stability estimates:

$$
\begin{aligned}
& \max _{0 \leq k \leq N}\left\|u_{k}^{h}\right\|_{L_{2 h}}+\max _{0 \leq k \leq N}\left\|u_{k}^{h}\right\|_{W_{2 h}^{1}} \leq M_{1}\left[\max _{1 \leq k \leq N-1}\left\|f_{k}^{h}\right\|_{L_{2 h}}\right. \\
& \left.+\left\|\psi^{h}\right\|_{L_{2 h}}+\left\|\varphi^{h}\right\|_{W_{2 h}^{1}}+\tau\left\|\varphi^{h}\right\|_{W_{2 h}^{2}}+\tau\left\|f_{1,1}^{h}\right\|_{L_{2 h}}\right], \\
& \max _{1 \leq k \leq N-1}\left\|\tau^{-2}\left(u_{k+1}^{h}-2 u_{k}^{h}+u_{k-1}^{h}\right)\right\|_{L_{2 h}}+\max _{0 \leq k \leq N}\left\|u_{k}^{h}\right\|_{W_{2 h}^{2}} \\
& \quad \leq M_{1}\left[\left\|f_{1}^{h}\right\|_{L_{2 h}}+\max _{2 \leq k \leq N-1}\left\|\tau^{-1}\left(f_{k}^{h}-f_{k-1}^{h}\right)\right\|_{L_{2 h}}\right. \\
& \left.+\left\|\psi^{h}\right\|_{W_{2 h}^{1}}+\left\|\varphi^{h}\right\|_{W_{2 h}^{2}}+\tau\left\|\varphi^{h}\right\|_{W_{2 h}^{3}}+\tau\left\|f_{1,1}^{h}\right\|_{W_{2 h}^{1}}\right] .
\end{aligned}
$$

Here $M_{1}$ does not depend on $\tau, h, \varphi^{h}(x), \psi^{h}(x), f_{1,1}^{h}$ and $f_{k}^{h}$, $1 \leq k<N$.

The proof of Theorem 4 is based on the proof of abstract Theorem 3 and the symmetry property of the operator $A_{h}^{x}$ defined by (35).

Second, let $\Omega$ be the unit open cube in the $m$-dimensional Euclidean space $\mathbb{R}^{m}\left\{x=\left(x_{1}, \ldots, x_{m}\right): 0<x_{j}<1,1 \leq j \leq\right.$ $m\}$ with boundary $S, \bar{\Omega}=\Omega \cup S$. In $[0,1] \times \Omega$, the mixed boundary value problem for the multidimensional hyperbolic equation

$$
\begin{aligned}
& \frac{\partial^{2} u(t, x)}{\partial t^{2}}-\sum_{r=1}^{m}\left(a_{r}(x) u_{x_{r}}\right)_{x_{r}}=f(t, x), \\
& x=\left(x_{1}, \ldots, x_{m}\right) \in \Omega_{h}, \quad 0<t<1, \\
& u^{h}(0, x)=\sum_{j=1}^{n} \alpha_{j} u^{h}\left(\lambda_{j}, x\right)+\varphi^{h}(x), \quad x \in \bar{\Omega}_{h}, \\
& u_{t}^{h}(0, x)=\sum_{j=1}^{n} \beta_{j} u_{t}^{h}\left(\lambda_{j}, x\right)+\psi^{h}(x), \quad x \in \bar{\Omega}_{h}, \\
& u^{h}(t, x)=0, \quad x \in S
\end{aligned}
$$

is considered. Here $a_{r}(x),(x \in \Omega), \varphi(x), \psi(x)\left(x \in \bar{\Omega}_{h}\right)$, and $f(t, x)\left(t \in(0,1), x \in \Omega_{h}\right)$ are given smooth functions and $a_{r}(x) \geq a>0$.
We introduce the Hilbert space $L_{2}(\bar{\Omega})$ of all square integrable functions defined on $\bar{\Omega}$, equipped with the norm

$$
\|f\|_{L_{2}(\bar{\Omega})}=\left\{\int \cdots \int_{x \in \bar{\Omega}}|f(x)|^{2} d x_{1} \cdots d x_{m}\right\}^{1 / 2} .
$$

Problem (39) has a unique smooth solution $u(t, x)$, and $\varphi(x)$, $\psi(x), a_{r}(x)$, and $f(t, x)$ are smooth functions. This allows us to reduce the mixed problem (39) to multipoint NBVP (1) in a Hilbert space $H=L_{2}(\bar{\Omega})$ with a self-adjoint positive definite operator $A^{x}$ defined by (39).

The discretization of problem (39) is carried out in two steps. In the first step, we define the grid sets

$$
\begin{aligned}
\widetilde{\Omega}_{h} & =\left\{x=x_{r}=\left(h_{1} r_{1}, \ldots, h_{m} r_{m}\right), r=\left(r_{1}, \ldots, r_{m}\right), 0\right. \\
& \left.\leq r_{j} \leq N_{j}, h_{j} N_{j}=1, j=1, \ldots, m\right\}, \\
\Omega_{h} & =\widetilde{\Omega}_{h} \cap \Omega, \\
S_{h} & =\widetilde{\Omega}_{h} \cap S .
\end{aligned}
$$

We introduce the Banach space $L_{2 h}=L_{2}\left(\widetilde{\Omega}_{h}\right), W_{2 h}^{1}=$ $W_{2 h}^{1}\left(\widetilde{\Omega}_{h}\right)$, and $W_{2 h}^{2}=W_{2 h}^{2}\left(\widetilde{\Omega}_{h}\right)$ of the grid functions $\varphi^{h}(x)=$ $\left\{\varphi\left(h_{1} r_{1}, \ldots, h_{m} r_{m}\right)\right\}$ defined on $\widetilde{\Omega}_{h}$, equipped with norms

$$
\begin{aligned}
\left\|\varphi^{h}\right\|_{L_{2}\left(\widetilde{\Omega}_{h}\right)}= & \left(\sum_{x \in \bar{\Omega}_{h}}\left|\varphi^{h}(x)\right|^{2} h_{1} \cdots h_{m}\right)^{1 / 2}, \\
\left\|\varphi^{h}\right\|_{W_{2 h}^{1}}= & \left\|\varphi^{h}\right\|_{L_{2 h}} \\
& +\left(\sum_{x \in \bar{\Omega}_{h}} \sum_{r=1}^{m}\left|\left(\varphi^{h}\right)_{\bar{x}_{r}, j_{r}}\right|^{2} h_{1} \cdots h_{m}\right)^{1 / 2}, \\
\left\|\varphi^{h}\right\|_{W_{2 h}^{2}}= & \left\|\varphi^{h}\right\|_{L_{2 h}} \\
& +\left(\sum_{x \in \bar{\Omega}_{h}} \sum_{r=1}^{m}\left|\left(\varphi^{h}\right)_{\bar{x}_{r}}\right|^{2} h_{1} \cdots h_{m}\right)^{1 / 2} \\
& +\left(\sum_{x \in \bar{\Omega}_{h}} \sum_{r=1}^{m}\left|\left(\varphi^{h}\right)_{x_{r} \bar{x}_{r}, j_{r}}\right|^{2} h_{1} \cdots h_{m}\right)^{1 / 2},
\end{aligned}
$$

respectively. To the differential operator $A$ generated by problem (39), we assign the difference operator $A_{h}^{x}$ by the formula

$$
A_{h}^{x} u_{x}^{h}=-\sum_{r=1}^{m}\left(a_{r}(x) u_{\bar{x}_{r}}^{h}\right)_{x_{r}, j_{r}}
$$

acting in the space of grid functions $u^{h}(x)$, satisfying the conditions $u^{h}(x)=0$ for all $x \in S_{h}$. It is known that $A_{h}^{x}$ is 
a self-adjoint positive definite operator in $L_{2}\left(\widetilde{\Omega}_{h}\right)$. With the help of $A_{h}^{x}$ we arrive at the multipoint NBVP:

$$
\begin{array}{r}
\frac{d^{2} v^{h}(t, x)}{d t^{2}}+A_{h}^{x} v^{h}(t, x)=f^{h}(t, x), \\
0<t<1, \quad x \in \Omega_{h}, \\
v^{h}(0, x)=\sum_{j=1}^{n} \alpha_{j} v^{h}\left(\lambda_{j}, x\right)+\varphi^{h}(x), \\
x \in \widetilde{\Omega}_{h}, \\
\frac{d v^{h}(0, x)}{d t}=\sum_{j=1}^{n} \beta_{j} v_{t}^{h}\left(\lambda_{j}, x\right)+\psi^{h}(x), \\
x \in \widetilde{\Omega}_{h}
\end{array}
$$

for an infinite system of ordinary differential equations.

In the second step, we replace problem (44) by difference scheme (45):

$$
\begin{aligned}
& \tau^{-2}\left(u_{k+1}^{h}(x)-2 u_{k}^{h}(x)+u_{k-1}^{h}(x)\right)+\frac{2}{3} A_{h}^{x} u_{k}^{h}(x)+\frac{1}{6} \\
& \cdot A_{h}^{x}\left(u_{k+1}^{h}(x)+u_{k-1}^{h}(x)\right)+\frac{1}{12} \tau^{2}\left(A_{h}^{x}\right)^{2} u_{k+1}^{h}(x) \\
& =f_{k}^{h}(x), \\
& f_{k}^{h}(x)=\frac{2}{3} f^{h}\left(t_{k}, x\right)+\frac{1}{6}\left(f^{h}\left(t_{k+1}, x\right)+f^{h}\left(t_{k-1}, x\right)\right) \\
& -\frac{1}{12} \tau^{2}\left(-A f^{h}\left(t_{k+1}, x\right)+f_{t t}^{h}\left(t_{k+1}, x\right)\right), \\
& t_{k}=k \tau, 1 \leq k \leq N-1, N \tau=1, x \in \Omega_{h}, \\
& u_{0}^{h}(x)=\sum_{j=1}^{n} \alpha_{j}\left\{u_{\left[\lambda_{j} / \tau\right]}^{h}(x)\right. \\
& +\tau^{-1}\left(u_{\left[\lambda_{j} / \tau\right]}^{h}(x)-u_{\left[\lambda_{j} / \tau\right]-1}^{h}(x)\right)\left(\lambda_{j}-\left[\frac{\lambda_{j}}{\tau}\right] \tau\right) \\
& +\frac{3}{2}\left(f_{\left[\lambda_{j} / \tau\right]}-A_{h}^{x} u_{\left[\lambda_{j} / \tau\right]}^{h}(x)\right)\left(\lambda_{j}-\left[\frac{\lambda_{j}}{\tau}\right] \tau\right)^{2} \\
& +\frac{7}{6}\left(f_{\left[\lambda_{j} / \tau\right]}^{\prime}-\tau^{-1} A_{h}^{x}\left(u_{\left[\lambda_{j} / \tau\right]}^{h}(x)-u_{\left[\lambda_{j} / \tau\right]-1}^{h}(x)\right)\right) \\
& \left.\cdot\left(\lambda_{j}-\left[\frac{\lambda_{j}}{\tau}\right] \tau\right)^{3}\right\}+\varphi^{h}(x), \quad x \in \Omega_{h}, \\
& \left(I+\tau^{2}\left(A_{h}^{x}\right)^{4}\right) \tau^{-1}\left(u_{1}^{h}(x)-u_{0}^{h}(x)\right)=\sum_{j=1}^{n} \beta_{j} \\
& \cdot\left\{\tau^{-1}\left(u_{\left[\lambda_{j} / \tau\right]}^{h}(x)-u_{\left[\lambda_{j} / \tau\right]-1}^{h}(x)\right)\right.
\end{aligned}
$$

$$
\begin{aligned}
& +\left(f_{\left[\lambda_{j} / \tau\right]}-A_{h}^{x} u_{\left[\lambda_{j} / \tau\right]}^{h}(x)\right)\left(\lambda_{j}-\left[\frac{\lambda_{j}}{\tau}\right] \tau\right) \\
& +\frac{1}{2 !}\left(f_{\left[\lambda_{j} / \tau\right]}^{\prime}-\tau^{-1} A_{h}^{x}\left(u_{\left[\lambda_{j} / \tau\right]}^{h}(x)-u_{\left[\lambda_{j} / \tau\right]-1}^{h}(x)\right)\right) \\
& \cdot\left(\lambda_{j}-\left[\frac{\lambda_{j}}{\tau}\right] \tau\right)^{2} \\
& +\frac{1}{3 !}\left(f_{\left[\lambda_{j} / \tau\right]}^{\prime \prime}-A_{h}^{x} f_{\left[\lambda_{j} / \tau\right]}+\left(A_{h}^{x}\right)^{2} u_{\left[\lambda_{j} / \tau\right]}^{h}(x)\right) \\
& \left.\cdot\left(\lambda_{j}-\left[\frac{\lambda_{j}}{\tau}\right] \tau\right)^{3}\right\}+\psi^{h}(x), \quad x \in \Omega_{h}, \\
& f_{1,1}^{h}(x)=\frac{1}{2} f^{h}(0, x)+\frac{\tau}{6} f_{t}^{h}(0, x) .
\end{aligned}
$$

Theorem 5. Let $\tau$ and $|h|$ be sufficiently small numbers. Then, the solution of difference scheme (45) satisfies the following stability estimates:

$$
\begin{gathered}
\max _{0 \leq k \leq N}\left\|u_{k}^{h}\right\|_{L_{2 h}}+\max _{0 \leq k \leq N}\left\|u_{k}^{h}\right\|_{W_{2 h}^{1}} \leq M_{1}\left[\max _{1 \leq k \leq N-1}\left\|f_{k}^{h}\right\|_{L_{2 h}}\right. \\
\left.+\left\|\psi^{h}\right\|_{L_{2 h}}+\left\|\varphi^{h}\right\|_{W_{2 h}^{1}}+\tau\left\|\varphi^{h}\right\|_{W_{2 h}^{2}}+\tau\left\|f_{1,1}^{h}\right\|_{L_{2 h}}\right] \\
\max _{1 \leq k \leq N-1}\left\|\tau^{-2}\left(u_{k+1}^{h}-2 u_{k}^{h}+u_{k-1}^{h}\right)\right\|_{L_{2 h}}+\max _{0 \leq k \leq N}\left\|u_{k}^{h}\right\|_{W_{2 h}^{2}} \\
\quad \leq M_{1}\left[\left\|f_{1}^{h}\right\|_{L_{2 h}}+\max _{2 \leq k \leq N-1}\left\|\tau^{-1}\left(f_{k}^{h}-f_{k-1}^{h}\right)\right\|_{L_{2 h}}\right. \\
\left.+\left\|\psi^{h}\right\|_{W_{2 h}^{1}}+\left\|\varphi^{h}\right\|_{W_{2 h}^{2}}+\tau\left\|\varphi^{h}\right\|_{W_{2 h}^{3}}+\tau\left\|f_{1,1}^{h}\right\|_{W_{2 h}^{1}}\right] .
\end{gathered}
$$

Here $M_{1}$ does not depend on $\tau, h, \varphi^{h}(x), \psi^{h}(x), f_{1,1}^{h}$, and $f_{k}^{h}$, $1 \leq k<N$.

Proof of Theorem 5 is based on the proof of abstract Theorem 3 , the symmetry property of the operator $A_{h}^{x}$ defined by formula (43), and the following theorem on the coercivity inequality for the solution of the elliptic difference problem in $L_{2 h}$.

Theorem 6. For the solutions of the elliptic difference problem

$$
\begin{aligned}
A_{h}^{x} u^{h}(x) & =\omega^{h}(x), \quad x \in \Omega_{h}, \\
u^{h}(x) & =0, \quad x \in S_{h}
\end{aligned}
$$

the following coercivity inequality holds [25]:

$$
\sum_{r=1}^{m}\left\|u_{x_{r} \bar{x}_{r}, j_{r}}^{h}\right\|_{L_{2 h}} \leq M\left\|\omega^{h}\right\|_{L_{2 h}} .
$$

Here $M$ does not depend on $h, \omega^{h}(x)$. 
TABLE 1: Errors for the approximate solutions of problem (49).

\begin{tabular}{lccc}
\hline Method & $\begin{array}{l}N=20, \\
M=20\end{array}$ & $\begin{array}{l}N=40, \\
M=40\end{array}$ & $\begin{array}{l}N=80, \\
M=80\end{array}$ \\
\hline $\begin{array}{l}\text { Difference scheme } \\
\text { solution }\end{array}$ & 0,0005962 & 0,0001554 & 0,00003946 \\
\hline
\end{tabular}

\section{Numerical Results}

In this section some numerical results are presented. The multipoint NBVP

$$
\begin{aligned}
& \frac{\partial^{2} u(t, x)}{\partial t^{2}}-\frac{\partial^{2} u(t, x)}{\partial x^{2}}=\left(t^{2}-2 t+3\right) \sin x, \\
& 0<t<1,0<x<\pi, \\
& u(0, x)=\frac{1}{8} u(1, x)+\frac{1}{8} u\left(\frac{1}{2}, x\right)+\frac{31}{32} \sin x, \\
& 0 \leq x \leq \pi \\
& u_{t}(0, x)=\frac{1}{8} u_{t}(1, x)+\frac{1}{8} u_{t}\left(\frac{1}{2}, x\right)-\frac{15}{8} \sin x, \\
& 0 \leq x \leq \pi \\
& u(t, 0)=u(t, \pi)=0, \quad 0 \leq t \leq 1
\end{aligned}
$$

for one-dimensional hyperbolic equation is considered. The exact solution of (49) is

$$
u(t, x)=(1-t)^{2} \sin x
$$

For the approximate solution of problem (49) the third order of accuracy difference scheme (2) is used, and the set $[0,1]_{\tau} \times$ $[0, \pi]_{h}$ of a family of grid points depending on the small parameters $\tau$ and $h$ is considered: $[0,1]_{\tau} \times[0, \pi]_{h}=\left\{\left(t_{k}, x_{n}\right)\right.$ : $t_{k}=k \tau, 0 \leq k \leq N, N \tau=1, x_{n}=n h, 0 \leq n \leq M, M h=$ $\pi$. The implementations of the numerical experiments are carried out by Matlab.

The errors are computed by the following formula:

$$
E_{M}^{N}=\max _{1 \leq k \leq N-1,1 \leq k \leq M-1}\left|u\left(t_{k}, x_{n}\right)-u_{n}^{k}\right| .
$$

Here, $u\left(t_{k}, x_{n}\right)$ represents the exact solution and $u_{n}^{k}$ represents the numerical solution at $\left(t_{k}, x_{n}\right)$. Errors are presented in Table 1.

In the table, the results are presented for different $M$ and $N$ values which are the step numbers for time and space variables, respectively. The results in Table 1 indicate that error decreases as $M$ and $N$ values get larger. Therefore it can be verified by the experimental results (Table 1) that the difference scheme is stable.

\section{Conclusion}

In this paper a third order of accuracy unconditionally stable difference scheme for the approximate solution of hyperbolic multipoint NBVP in a Hilbert space with self-adjoint positive definite operator is presented. The stability is established without any assumptions in respect of the grid steps $\tau$ and $h$. Numerical results verifying the theoretical statements are presented. It is not easy to obtain arbitrary order stable difference schemes. Nevertheless one can obtain fourth order or higher order stable difference schemes using our results and method. It is possible to consider the same problem with fractional derivative or with small parameter $\varepsilon$.

\section{Conflict of Interests}

The authors declare that there is no conflict of interests regarding the publication of this paper.

\section{Acknowledgments}

The authors would like to thank Professor Dr. A. Ashyralyev and Professor Dr. P. E. Sobolevskii for their helpful suggestions to the improvement of this paper.

\section{References}

[1] H. Lamb, Hydrodynamics, Cambridge University Press, Cambridge, UK, 1993.

[2] G. Gallavotti, Foundations of Fluid Dynamics, Springer, 2002.

[3] T. Miloh, Mathematics Approaches in Hydrodynamics, Society for Industrial and Applied Mathematics, Philadelphia, Pa, USA, 1991.

[4] D. S. Jones, Acoustic and Electromagnetic Waves, Oxford Science Publications, The Clarendon Press/Oxford University Press, New York, NY, USA, 1986.

[5] A. Taflove, Computational Electrodynamics: The Finite-Difference Time-Domain Method, Artech House, Boston, Mass, USA, 1995.

[6] Z. Direk and M. Ashyraliyev, "FDM for the integral-differential equation of the hyperbolic type," Advances in Difference Equations, vol. 2014, article 132, 2014.

[7] Z. Direk and M. Ashyraliyev, "A second order of accuracy finite difference scheme for the integral-differential equation of the hyperbolic type," AIP Conference Proceedings, vol. 1611, no. 1, pp. 398-403, 2014, Proceedings of the International Conference on Analysis and Applied Mathematics.

[8] Z. Direk and M. Ashyraliyev, "Finite difference method for the integral-differential equation of the hyperbolic type," AIP Conference Proceedings, vol. 1470, no. 1, pp. 266-269, 2012, (International Conference on Analysis and Applied Mathematics Book Series).

[9] M. Ashyraliyev, "A note on the stability of the integral-differential equation of the hyperbolic type in a Hilbert space," Numerical Functional Analysis and Optimization, vol. 29, no. 7-8, pp. 750-769, 2008.

[10] N. Aggez and M. Ashyralyyewa, "Numerical solution of stochastic hyperbolic equations," Abstract and Applied Analysis, vol. 2012, Article ID 824819, 20 pages, 2012.

[11] B. Gustafsson, H.-O. Kreiss, and J. Oliger, Time Dependent Problems and Difference Methods, John Wiley \& Sons, New York, NY, USA, 1995.

[12] M. Sapagovas, "On stability of the finite difference schemes for a parabolic equations with nonlocal condition," Journal of Computer Applied Mathematics, vol. 1, no. 1, pp. 89-98, 2003. 
[13] A. Ashyralyev and P. E. Sobolevskii, New Difference Schemes for Partial Differential Equations, Operator Theory: Advances and Applications, vol. 148, Birkhäuser, Basel, Switzerland, 2004.

[14] A. Ashyralyev and O. Yildirim, "On the numerical solution of hyperbolic IBVP with high-order stable finite difference schemes," Boundary Value Problems, vol. 2013, 29 pages, 2013.

[15] O. Yildirim and M. Uzun, "On the numerical solutions of high order stable difference schemes for the hyperbolic multipoint nonlocal boundary value problems," Applied Mathematics and Computation, vol. 254, pp. 210-218, 2015.

[16] O. Yildirim and M. Uzun, "High order of accuracy stable difference schemes for the approximate solution of the multipoint NBVP for the hyperbolic equation," in International Conference on Analysis and Applied Mathematics (ICAAM '14), vol. 1611 of AIP Conference Proceedings, pp. 305-309, Shymkent, Kazakhstan, September 2014.

[17] A. Ashyralyev and P. E. Sobolevskii, "A note on the difference schemes for hyperbolic equations," Abstract and Applied Analysis, vol. 6, no. 2, pp. 63-70, 2001.

[18] A. Ashyralyev and P. E. Sobolevskii, "Two new approaches for construction of the high order of accuracy difference schemes for hyperbolic differential equations," Discrete Dynamics in Nature and Society, vol. 2005, no. 2, pp. 183-213, 2005.

[19] A. Ashyralyev and O. Yildirim, "On multipoint nonlocal boundary value problems for hyperbolic differential and difference equations," Taiwanese Journal of Mathematics, vol. 14, no. 1, pp. 165-194, 2010.

[20] M. E. Koksal, "Recent developments on operator-difference schemes for solving nonlocal BVPs for the wave equation," Discrete Dynamics in Nature and Society, vol. 2011, Article ID 210261, 14 pages, 2011.

[21] O. Gercek, "Well-posedness of the first order of accuracy difference scheme for elliptic-parabolic equations in Hölder spaces," Abstract and Applied Analysis, vol. 2012, Article ID 237657, 12 pages, 2012.

[22] Y. Ozdemir and M. Kucukunal, "A note on nonlocal boundary value problems for hyperbolic Schrödinger equations," Abstract and Applied Analysis, vol. 2012, Article ID 687321, 12 pages, 2012.

[23] T. E. Simos and P. S. Williams, "A finite-difference method for the numerical solution of the Schrödinger equation," Journal of Computational and Applied Mathematics, vol. 79, no. 2, pp. 189205, 1997.

[24] P. E. Sobolevskii and L. M. Chebotaryeva, "Approximate solution by method of lines of the Cauchy problem for an abstract hyperbolic equations," Izvestiya Vysshikh Uchebnykh Zavedenii. Matematika, no. 5, pp. 103-116, 1977 (Russian).

[25] P. E. Sobolevskii, Difference Methods for the Approximate Solution of Differential Equations, Izdat Voronezh Gosud University, Voronezh, Russia, 1975.

[26] A. R. Mitchell and D. F. Griffiths, The Finite Difference Method in Partial Differential Equations, Wiley, New York, NY, USA, 1980.

[27] P. D. Lax and B. Wendroff, "Difference schemes for hyperbolic equations with high order of accuracy," Communications on Pure and Applied Mathematics, vol. 17, pp. 381-398, 1964.

[28] A. A. Samarskii, R. D. Lazarov, and V. L. Makarov, Difference Schemes for Differential Equations with Generalized Solutions, Vysshaya Shkola, Moscow, Russia, 1987, (Russian).

[29] H. O. Fattorini, Second Order Linear Differential Equations in Banach Spaces, vol. 108 of North-Holland Mathematics Studies, North-Holland, Amsterdam, The Netherlands, 1985.
[30] S. G. Krein, Linear Differential Equations in Banach Space, vol. 29 of Translations of Mathematical Monographs, American Mathematical Society, Providence, RI, USA, 1971, translated from the Russian by J. M. Danskin.

[31] S. Piskarev and Y. Shaw, "On certain operator families related to cosine operator function," Taiwanese Journal of Mathematics, vol. 1, no. 4, pp. 3585-3592, 1997. 


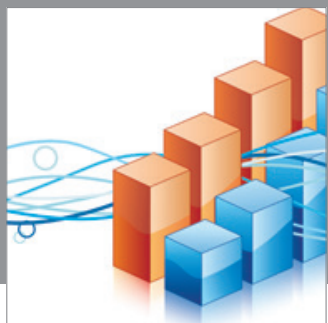

Advances in

Operations Research

mansans

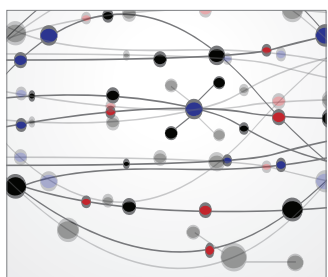

The Scientific World Journal
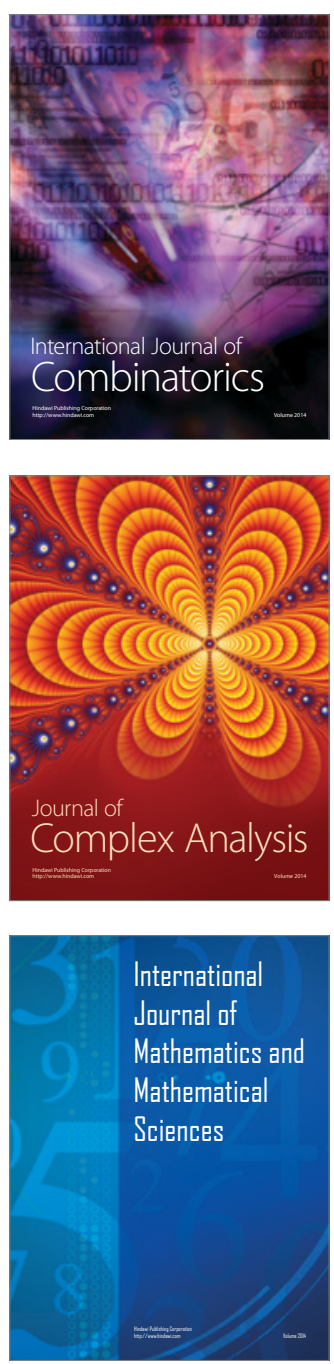
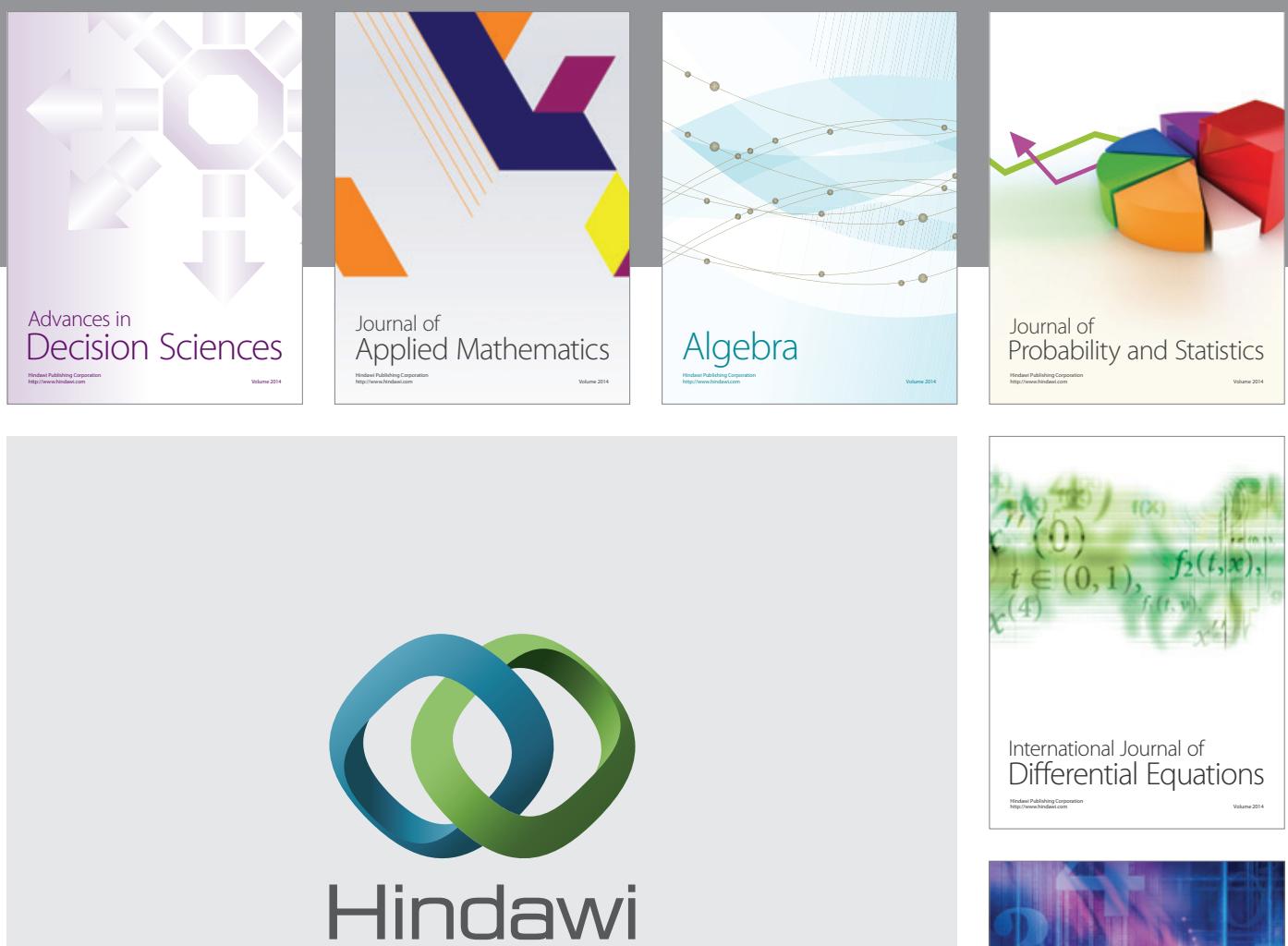

Submit your manuscripts at http://www.hindawi.com
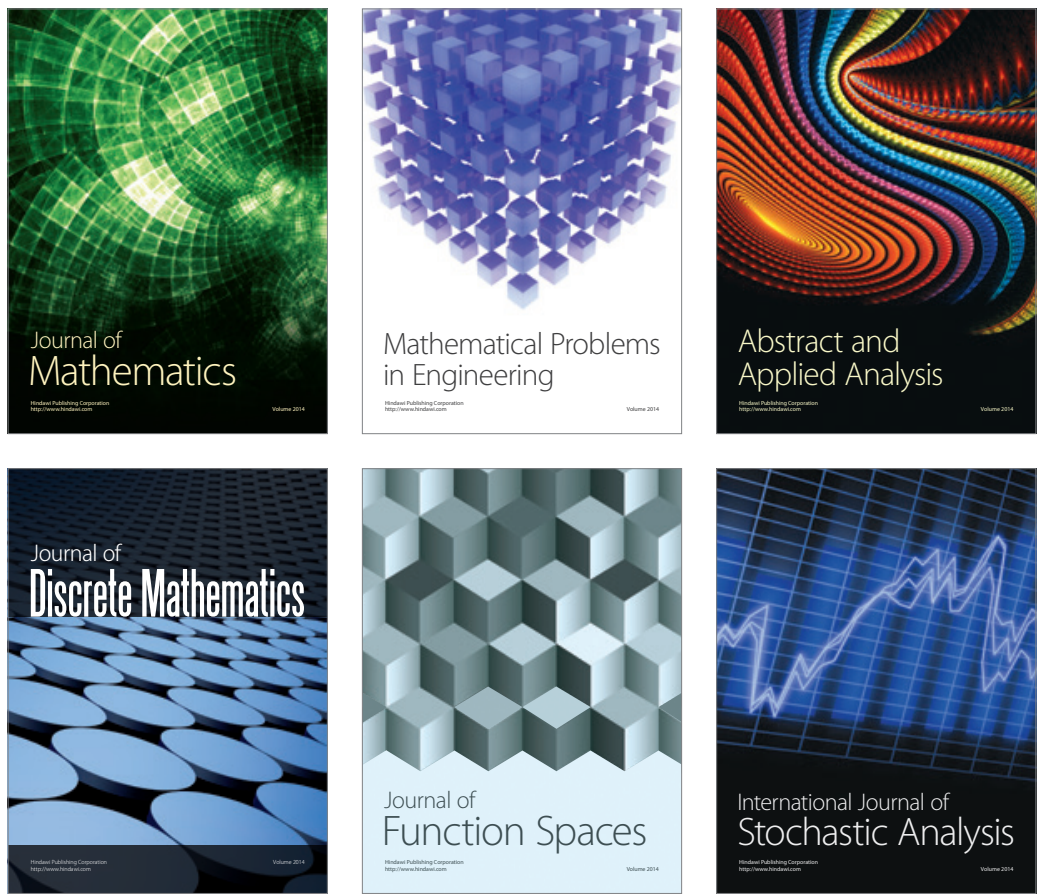

Journal of

Function Spaces

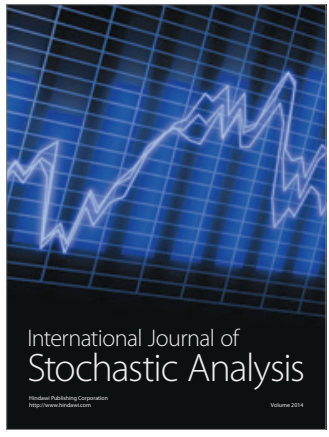

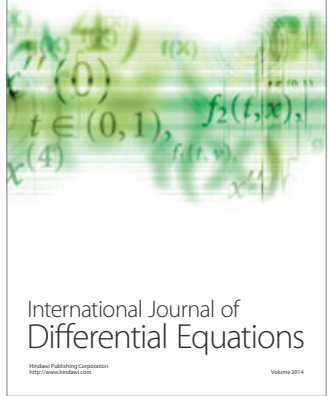
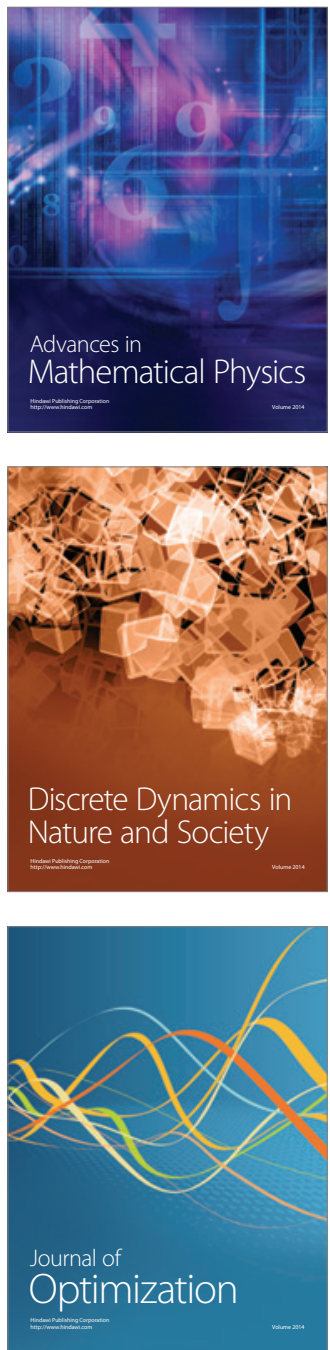